\title{
Behavioral Portfolio Selection in Continuous Time*
}

\author{
Hanqing Jin ${ }^{\dagger}$ and Xun Yu Zhou
}

February 11, 2013

\begin{abstract}
This paper formulates and studies a general continuous-time behavioral portfolio selection model under Kahneman and Tversky's (cumulative) prospect theory, featuring S-shaped utility (value) functions and probability distortions. Unlike the conventional expected utility maximization model, such a behavioral model could be easily misformulated (a.k.a. ill-posed) if its different components do not coordinate well with each other. Certain classes of an ill-posed model are identified. A systematic approach, which is fundamentally different from the ones employed for the utility model, is developed to solve a well-posed model, assuming a complete market and general Itô processes for asset prices. The optimal terminal wealth positions, derived in fairly explicit forms, possess surprisingly simple structure reminiscent of a gambling policy betting on a good state of the world while accepting a fixed, known loss in case of a bad one. An example with a two-piece CRRA utility is presented to illustrate the general results obtained, and is solved completely for all admissible parameters. The effect of the behavioral criterion on the risky allocations is finally discussed.
\end{abstract}

Key words: Portfolio selection, continuous time, cumulative prospect theory, behavioral criterion, ill-posedness, S-shaped function, probability distortion, Choquet integral

*This paper has benefited from comments of participants at the Quantitative Methods in Finance 2005 Conference in Sydney, the 2005 International Workshop on Financial Engineering and Risk Management in Beijing, the 2006 International Symposium on Stochastic Processes and Applications to Mathematical Finance in Ritsumeikan University, and the 2006 Workshop on Mathematical Finance and Insurance in Lijiang; and from the comments of Knut Aase, Andrew Cairns, Mark Davis, Peter Imkeller, Jacek Krawczyk, Terry Lyons, James Mirrlees, Eckhard Platen, Sheldon Ross, Larry Samuelson, Martin Schweizer, John van der Hoek, and David Yao. The authors thank especially Jia-an Yan for remarks and discussions over the years on the general topic considered in this paper. Two anonymous referees have given constructive comments leading to a much improved version. All errors are the responsibility of the authors. Zhou gratefully acknowledges financial support from the RGC Earmarked Grants CUHK4175/03E and CUHK418605, and the Croucher Senior Research Fellowship.

${ }^{\dagger}$ Department of Mathematics, National University of Singapore, Singapore. Email: $<$ matjinh@nus.edu.sg $>$.

${ }^{\ddagger}$ Mathematical Institute, University of Oxford, 24-29 St Giles’, Oxford, OX1 3LB, UK, and Department of Systems Engineering and Engineering Management, The Chinese University of Hong Kong, Shatin, Hong Kong. email: <zhouxy@maths.ox.ac.uk>. 


\section{Introduction}

Mean-variance and expected utility maximization are by far the two predominant investment decision rules in financial portfolio selection. Portfolio theory in the dynamic setting (both discrete time and continuous time) has been established in the past twenty years, again centering around these two frameworks while employing heavily among others the martingale theory, convex duality and stochastic control; see Duffie (1996), Karatzas and Shreve (1998), and Föllmer and Schied (2002) for systematic accounts on dynamic utility maximization, and Li and Ng (2000), Zhou and Li (2000), and Jin, Yan and Zhou (2004) for recent studies on the mean-variance (including extensions to mean-risk) counterpart.

Expected utility theory (EUT), developed by von Neumann and Morgenstern (1944) based on an axiomatic system, has an underlying assumption that decision makers are rational and risk averse when facing uncertainties. In the context of asset allocations, its basic tenets are: Investors evaluate wealth according to final asset positions; they are uniformly risk averse; and they are able to objectively evaluate probabilities. These, however, have long been criticized to be inconsistent with the way people do decision making in the real world. Substantial experimental evidences have suggested a systematic violation of the EUT principles. Specifically, the following anomalies (as opposed to the assumed rationality in EUT) in human behaviors are evident from daily life:

- People evaluate assets on gains and losses (which are defined with respect to a reference point), not on final wealth positions;

- People are not uniformly risk averse: they are risk-averse on gains and risk-taking on losses, and significantly more sensitive to losses than to gains;

- People overweight small probabilities and underweight large probabilities.

In addition, there are widely known paradoxes and puzzles that EUT fails to explain, including the Allais paradox [Allais (1953)], Ellesberg paradox [Ellesberg (1961)], Friedman and Savage puzzle [Friedman and Savage (1948)], and the equity premium puzzle [Mehra and Prescott (1985)].

Considerable attempts and efforts have been made to address the drawback of EUT, among them notably the so-called non-additive utility theory [see, for example, Fishburn (1998)]. Unfortunately, most of these theories are far too complicated to be analyzable and applicable, and some of them even lead to new paradoxes. In 1970s, Kahneman and Tversky (1979) proposed the prospect theory (PT) for decision making under uncertainty, incorporating

human emotions and psychology into their theory. Later, Tversky and Kahneman (1992) fine tuned the PT to the cumulated prospect theory (CPT) in order to be consistent with the firstorder stochastic dominance. Among many other ingredients, the key elements of Kahneman and Tversky's Nobel-prize-winning theory are

- A reference point (or neutral outcome/benchmark/breakeven point/status quo) in wealth that defines gains and losses; 
- A value function (which replaces the notion of utility function), concave for gains and convex for losses, and steeper for losses than for gains (a behavior called loss aversion);

- A probability distortion that is a nonlinear transformation of the probability scale, which enlarges a small probability and diminishes a large probability.

There have been burgeoning research interests in incorporating the PT into portfolio choice; nonetheless they have been hitherto overwhelmingly limited to the single-period setting; see for example Benartzi and Thaler (1995), Shefrin and Statman (2000), Levy and Levy (2004), Bassett et al. (2004), Gomes (2005), and De Giorgi and Post (2005), with emphases on qualitative properties and empirical experiments. Analytical research on dynamic, especially continuous-time, asset allocation featuring behavioral criteria is literally nil according to our best knowledge. [In this connection the only paper we know of that has some bearing on the PT for the continuous time setting is Berkelaar, Kouwenberg and Post (2004) where a very specific two-piece power utility function is considered; however, the probability distortion, which is one of the major ingredients of the PT and which causes the main difficulty, is absent in that paper.] Such a lack of study on continuous-time behavioral portfolio selection is certainly not because the problem is uninteresting or unimportant; rather it is because, we believe, that the problem is massively difficult as compared with the conventional expected utility maximization model. Many conventional and convenient approaches, such as convex optimization, dynamic programming, and stochastic control, fall completely apart in handling such a behavioral model: First, the utility function (or value function as called in the PT) is partly concave and partly convex (also referred to as an $S$-shaped function), whereas the global convexity/concavity is a necessity in traditional optimization. Second, the nonlinear distortion in probabilities abolishes virtually all the nice properties associated with the normal additive probability and linear expectation. In particular, the dynamic consistency of the conditional expectation with respect to a filtration, which is the foundation of the dynamic programming principle, is absent due to the distorted probability. Worse still, the coupling of these two ill-behaved features greatly amplifies the difficulty of the problem ${ }^{1}$. Even the well-posedness of the problem ${ }^{2}$ is no longer something that can be taken for granted.

This paper first establishes a general continuous-time portfolio selection model under the CPT, involving behavioral criteria defined on possibly continuous random variables. The probability distortions lead to the involvement of the Choquet integrals [Choquet (1953/54)], instead of the conventional expectation. We then carry out, analytically, extensive investigations on the model while developing new approaches in deriving the optimal solutions. First of all, by assuming that the market is complete, the asset prices follow general Itô processes, and the individual behavior of the investor in question will not affect the market, we need only to consider an optimization problem in terms of the terminal wealth. This is the usual

\footnotetext{
${ }^{1}$ In Berkelaar, Kouwenberg and Post (2004) an essentially convexification technique is employed to deal with the non-convexity of the problem. However, it does not work any longer in the presence of a distorted probability.

${ }^{2} \mathrm{~A}$ maximization problem is called well-posed if its supremum is finite; otherwise it is ill-posed. An ill-posed problem is a mis-formulated one: the trade-off is not set right so that one can always push the objective value to be arbitrarily high.
} 
trick employed in the conventional utility maximization, which also enables us to get around the inapplicability of the dynamic programming in the current setting. Having said this, our main endeavor is to find the optimal terminal wealth, which is a fundamentally different and difficult problem due to the behavioral criterion. As mentioned earlier such a behavioral model could be easily ill-posed and, therefore, we first identify several general cases where the model is indeed ill-posed. Then we move on to finding optimal solutions for a well-posed model. In doing so we decompose the original problem into two sub-problems: one takes care of the gain part and the other the loss part, both parameterized by an initial budget that is the price of the gain part (i.e., the positive change) of the terminal payoff over the reference wealth position and an event when the terminal payoff represents a gain. At the outset the gain part problem is a constrained non-concave maximization problem due to the probability distortion; yet by changing the decision variable and taking a series of transformations, we turn it into a concave maximization problem where the Lagrange method is applicable. The loss part problem, nevertheless, is more subtle because it is to minimize a concave functional even after the similar transformations. We are able to characterize explicitly its solutions to be certain "corner points" via delicate analysis. There is yet one more twist in deriving the optimal solution to the original model given the solutions to the above two problems: one needs to find the "best" parameters - the initial budget and the event of a terminal gain by solving another constrained optimization problem.

As mathematically complicated and sophisticated the solution procedure turns out to be, the final solutions are surprisingly and beautifully simple: the optimal terminal wealth resembles the payoff of a portfolio of two binary (or digital) options written on a mutual fund (induced by the state pricing density), characterized by a single number. This number, in turn, can be identified by solving a very simple two-dimensional mathematical programming problem. The optimal strategy is therefore a gambling policy, betting on good states of the market, by buying a contingent claim and selling another. We present an example with the same value function taken by Tversky and Kahneman (1992), and demonstrate that our general results lead to a complete solution of the model for all admissible parameters involved. Furthermore, for the case when the market parameters are constants, we are able to derive the optimal portfolio in closed form, thereby understand how the behavioral criteria may change the risky asset allocations.

To summarize, the main contributions of this paper are: 1) we establish, for the first time, a bona fide continuous-time behavioral portfolio selection model à la cumulative prospect theory, featuring very general S-shaped utility functions and probability distortions; 2) we demonstrate that the well-posedness becomes an eminent issue for the behavioral model, and identify several ill-posed problems; 3) we develop an approach, fundamentally different from the existing ones for the expected utility model, to overcome the immense difficulties arising from the analytically ill-behaved utility functions and probability distortions. Some of the sub-problems solvable by this approach, such as constrained maximization and minimization of Choquet integrals, are interesting, in both theory and applications, in their own rights; and 4) we obtain fairly explicit solutions to a general model, and closed-form solutions for an important special case, based on which we are able to examine how the allocations to equity are influenced by behavioral criteria. 
The rest of the paper is organized as follow. In Section 2 the behavioral model is formulated, and its possible ill-posedness is addressed in Section 3. The main results of the paper are stated in Section 4. The procedure of analytically solving the general model is developed in Sections $5-7$, leading to a proof of the main results in Section 8. A special case with a two-piece CRRA utility function is presented in Section 9 to demonstrate the general results obtained. Section 10 addresses the issue of how the behavioral criterion would affect the risky allocations. Some concluding remarks are given in Section 11. Finally, technical preliminaries are relegated to an appendix.

\section{The Model}

In this paper $T$ is a fixed terminal time and $\left(\Omega, \mathcal{F}, P,\left\{\mathcal{F}_{t}\right\}_{t \geq 0}\right)$ is a fixed filtered complete probability space on which is defined a standard $\mathcal{F}_{t}$-adapted $m$-dimensional Brownian motion $W(t) \equiv\left(W^{1}(t), \cdots, W^{m}(t)\right)^{\prime}$ with $W(0)=0$. It is assumed that $\mathcal{F}_{t}=\sigma\{W(s): 0 \leq s \leq t\}$, augmented by all the null sets. Here and throughout the paper $A^{\prime}$ denotes the transpose of a matrix $A$.

We define a continuous-time financial market following Karatzas and Shreve (1998). In the market there are $m+1$ assets being traded continuously. One of the assets is a bank account whose price process $S_{0}(t)$ is subject to the following equation:

$$
d S_{0}(t)=r(t) S_{0}(t) d t, \quad t \in[0, T] ; \quad S_{0}(0)=s_{0}>0,
$$

where the interest rate $r(\cdot)$ is an $\mathcal{F}_{t}$-progressively measurable, scalar-valued stochastic process with $\int_{0}^{T}|r(s)| d s<+\infty$, a.s.. The other $m$ assets are stocks whose price processes $S_{i}(t)$, $i=1, \cdots, m$, satisfy the following stochastic differential equation (SDE):

$$
d S_{i}(t)=S_{i}(t)\left[b_{i}(t) d t+\sum_{j=1}^{m} \sigma_{i j}(t) d W^{j}(t)\right], \quad t \in[0, T] ; \quad S_{i}(0)=s_{i}>0,
$$

where $b_{i}(\cdot)$ and $\sigma_{i j}(\cdot)$, the appreciation and dispersion (or volatility) rates, respectively, are scalar-valued, $\mathcal{F}_{t}$-progressively measurable stochastic processes with $\int_{0}^{T}\left[\sum_{i=1}^{m}\left|b_{i}(t)\right|+\right.$ $\left.\sum_{i, j=1}^{m}\left|\sigma_{i j}(t)\right|^{2}\right] d t<+\infty$, a.s..

Set the excess rate of return vector process

$$
B(t):=\left(b_{1}(t)-r(t), \cdots, b_{m}(t)-r(t)\right)^{\prime},
$$

and define the volatility matrix process $\sigma(t):=\left(\sigma_{i j}(t)\right)_{m \times m}$. Basic assumptions imposed on the market parameters throughout this paper are summarized as follows:

\section{Assumption 2.1}

(i) There exists $c \in \mathbb{R}$ such that $\int_{0}^{T} r(s) d s \geq c$, a.s..

(ii) $\operatorname{Rank}(\sigma(t))=m$, a.e.t $\in[0, T]$, a.s.. 
(ii) There exists an $\mathbb{R}^{m}$-valued, uniformly bounded, $\mathcal{F}_{t}$-progressively measurable process $\theta(\cdot)$ such that $\sigma(t) \theta(t)=B(t)$, a.e. $t \in[0, T]$, a.s..

It is well known that under these assumptions there exists a unique risk-neutral (martingale) probability measure $Q$ defined by $\left.\frac{d Q}{d P}\right|_{\mathcal{F}_{t}}=\rho(t)$, where

$$
\rho(t):=\exp \left\{-\int_{0}^{t}\left[r(s)+\frac{1}{2}|\theta(s)|^{2}\right] d s-\int_{0}^{t} \theta(s)^{\prime} d W(s)\right\}
$$

is the pricing kernel or state density price. Denote $\rho:=\rho(T)$. It is clear that $0<\rho<+\infty$ a.s., and $0<E \rho<+\infty$.

A random variable $\xi$ is said to have no atom if $P\{\xi=a\}=0 \forall a \in \mathbb{R}$. The following assumption is in force throughout this paper.

Assumption $2.2 \rho$ admits no atom.

The preceding assumption is not essential, and is imposed to avoid undue technicality. In particular, it is satisfied when $r(\cdot)$ and $\theta(\cdot)$ are deterministic with $\int_{0}^{T}|\theta(t)|^{2} d t \neq 0$ (in which case $\rho$ is a nondegenerate lognormal random variable). We are also going to use the following notation:

$$
\begin{aligned}
& \bar{\rho} \equiv \operatorname{esssup} \rho:=\sup \{a \in \mathbb{R}: P\{\rho>a\}>0\} \\
& \underline{\rho} \equiv \operatorname{essinf} \rho:=\inf \{a \in \mathbb{R}: P\{\rho<a\}>0\}
\end{aligned}
$$

Consider an agent, with an initial endowment $x_{0} \in \mathbb{R}$ (fixed throughout this paper) ${ }^{3}$, whose total wealth at time $t \geq 0$ is denoted by $x(t)$. Assume that the trading of shares takes place continuously in a self-financing fashion (i.e., there is no consumption or income) and there are no transaction costs. Then $x(\cdot)$ satisfies [see, e.g., Karatzas and Shreve (1998)]

$$
d x(t)=\left[r(t) x(t)+B^{\prime}(t) \pi(t)\right] d t+\pi(t)^{\prime} \sigma(t) d W(t), \quad t \in[0, T] ; \quad x(0)=x_{0},
$$

where $\pi(\cdot) \equiv\left(\pi_{1}(\cdot), \cdots, \pi_{m}(\cdot)\right)^{\prime}$ is the portfolio of the agent with $\pi_{i}(t), \quad i=1,2 \cdots, m$, denoting the total market value of the agent's wealth in the $i$-th asset at time $t$. A portfolio $\pi(\cdot)$ is said to be admissible if it is an $\mathbb{R}^{m}$-valued, $\mathcal{F}_{t}$-progressively measurable process with

$$
\int_{0}^{T}\left|\sigma(t)^{\prime} \pi(t)\right|^{2} d t<+\infty \text { and } \int_{0}^{T}\left|B(t)^{\prime} \pi(t)\right| d t<+\infty, \quad \text { a.s.. }
$$

An admissible portfolio $\pi(\cdot)$ is said to be tame if the corresponding discounted wealth process, $S_{0}(t)^{-1} x(t)$, is almost surely bounded from below (the bound may depend on $\pi(\cdot)$ ).

The following result follows from Karatzas and Shreve (1998, p. 24, Theorem 6.6) noting Karatzas and Shreve (1998, p. 21, Definition 6.1) or Cox and Huang (1989).

Proposition 2.1 For any $\mathcal{F}_{T}$-measurable random variable $\xi$ such that $\xi$ is almost surely bounded from below and $E[\rho \xi]=x_{0}$, there exists a tame admissible portfolio $\pi(\cdot)$ such that the corresponding wealth process $x(\cdot)$ satisfies $x(T)=\xi$.

\footnotetext{
${ }^{3}$ Precisely speaking, $x_{0}$ should be the difference between the agent's initial wealth and a (discounted) reference wealth; for details see Remarks 2.1 and 2.2 below.
} 
In the conventional portfolio theory, an investor's preference is modelled by the expected utility of the terminal wealth. In this paper, we study a portfolio model featuring human behaviors by working within the CPT framework of Tversky and Kahneman (1992). First of all, in CPT there is a natural outcome or benchmark, assumed to be 0 (evaluated at the terminal time, $T$ ) in this paper without loss of generality (see Remark 2.1 below for elaborations on this point), which serves as a base point to distinguish gains from losses. Next, we are given two utility functions $u_{+}(\cdot)$ and $u_{-}(\cdot)$, both mapping from $\mathbb{R}^{+}$to $\mathbb{R}^{+}$, that measure the gains and losses respectively. There are two additional functions $T_{+}(\cdot)$ and $T_{-}(\cdot)$ from $[0,1]$ to $[0,1]$, representing the distortions in probability for the gains and losses respectively. The technical assumptions on these functions, which will be imposed throughout this paper, are summarized as follows.

Assumption $2.3 u_{+}(\cdot)$ and $u_{-}(\cdot): \mathbb{R}^{+} \mapsto \mathbb{R}^{+}$, are strictly increasing, concave, with $u_{+}(0)=$ $u_{-}(0)=0$. Moreover, $u_{+}(\cdot)$ is strictly concave and twice differentiable, with the Inada conditions $u_{+}^{\prime}(0+)=+\infty$ and $u_{+}^{\prime}(+\infty)=0$.

Assumption $2.4 T_{+}(\cdot)$ and $T_{-}(\cdot):[0,1] \mapsto[0,1]$, are differentiable and strictly increasing, with $T_{+}(0)=T_{-}(0)=0$ and $T_{+}(1)=T_{-}(1)=1$.

Now, given a contingent claim (a random variable) $X$, we assign it a value $V(X)$ by

$$
V(X)=V_{+}\left(X^{+}\right)-V_{-}\left(X^{-}\right)
$$

where

$$
V_{+}(Y):=\int_{0}^{+\infty} T_{+}\left(P\left\{u_{+}(Y)>y\right\}\right) d y, \quad V_{-}(Y):=\int_{0}^{+\infty} T_{-}\left(P\left\{u_{-}(Y)>y\right\}\right) d y
$$

for any random variable $Y \geq 0$, a.s.. (Throughout this paper $a^{+}$and $a^{-}$denote respectively the positive and negative parts of a real number a.) It is evident that both $V_{+}$and $V_{-}$are non-decreasing in the sense that $V_{ \pm}(X) \geq V_{ \pm}(Y)$ for any random variables $X$ and $Y$ with $X \geq Y$ a.s. . Moreover, $V_{+}(x)=u_{+}(x)$ and $V_{-}(x)=u_{-}(x) \forall x \in \mathbb{R}^{+}$. Finally, $V$ is also non-decreasing.

If $T_{+}(x)=x$ (there is no distortion) then $V_{+}(Y)=E\left[u_{+}(Y)\right]$ (likewise with $V_{-}$); hence $V_{+}$is a generalization of the expected utility. Yet this generalization poses a fundamentally different (and difficult) feature, namely, the set function $T_{+} \circ P$ is a capacity [Choquet $(1953 / 54)$ ] which is a non-additive measure as opposed to the standard notion of probability. So the definition of $V_{+}$involves the so-called Choquet integral [see Denneberg (1994) for a comprehensive account on Choquet integrals]. Notice that with the Choquet integral the dynamic consistency of conditional expectation, which is the base for the dynamic programming principle, is lost ${ }^{4}$.

In CPT the utility (or value) function $u(\cdot)$ is given on the whole real line, which is convex on $\mathbb{R}^{-}$and concave on $\mathbb{R}^{+}$(corresponding to the observation that people tend to be risk-averse

\footnotetext{
${ }^{4}$ The dynamic consistency refers to the following equality: $E\left(E\left(X \mid \mathcal{F}_{t}\right) \mid \mathcal{F}_{s}\right)=E\left(X \mid \mathcal{F}_{s}\right)$ if $\mathcal{F}_{s} \subseteq \mathcal{F}_{t}$. The problem of generalizing conditional expectation to Choquet integral remains largely open, not to mention the validity of the corresponding dynamic consistency in any sense; see Denneberg (1994, Chapter 12).
} 
on gains and risk-seeking on losses). Such a function is said to be of $S$-shaped. In our model, we separate the utility on gains and losses by letting $u_{+}(x):=u(x)$ and $u_{-}(x)=-u(-x)$ whenever $x \geq 0$. Thus our model is equivalent to the one with an overall S-shaped utility function.

In our model, the value $V(X)$ is defined on a general random variable $X$, possibly a continuous one, which is necessary for the continuous-time portfolio selection model, as opposed to Tversky and Kahneman (1992) where only discrete random variables are treated. Moreover, our definition of $V$ agrees with that in Tversky and Kahneman (1992) if $X$ is discrete [see Tversky and Kahneman (1992, pp. 300-301)].

Under this CPT framework, our portfolio selection problem is to find the most preferable portfolios, in terms of maximizing the value $V(x(T))$, by continuously managing the portfolio. The mathematical formulation is as follows:

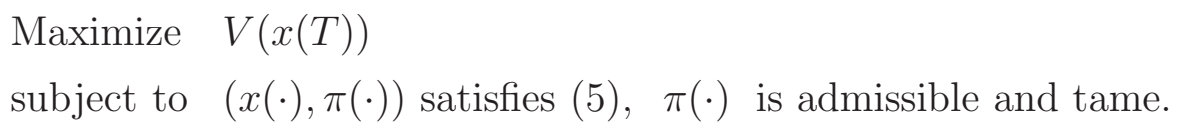

In view of Proposition 2.1, in order to solve (6) one needs only first to solve the following optimization problem in the terminal wealth, $X$ :

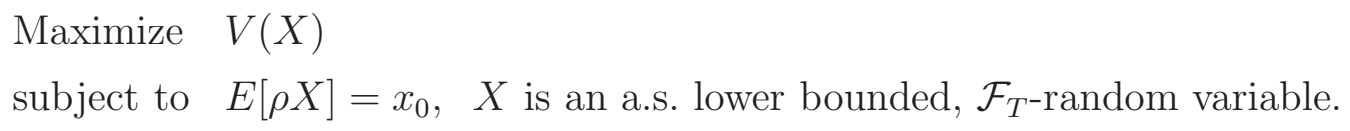

Once (7) is solved with a solution $X^{*}$, the optimal portfolio is then the one replicating $X^{*}$ (as determined by Proposition 2.1). Therefore, in the rest of the paper we will focus on Problem (7). Recall that a maximization problem is called well-posed if the supremum of its objective is finite; otherwise it is called ill-posed. One tries to find an optimal solution only if the problem is known a priori to be well-posed.

REMARK 2.1 If the reference point at $T$ is a general $\mathcal{F}_{T}$-measurable random variable $\xi$ (instead of 0 ), then, since the market is complete, we can replicate $\xi$ by a replicating portfolio $\bar{\pi}(\cdot)$ with the corresponding wealth process $\bar{x}(\cdot)$. [Incidentally, one can also take this case as one where there is a dynamically and stochastically changing reference trajectory $\bar{x}(\cdot)$.] In this case, by considering $x(t)-\bar{x}(t)$ as the state variable the problem (6) is reduced to one with the reference point being 0 . [In view of this, the process $x(\cdot)$ determined by (5) actually represents the magnitude of the change in wealth from the the price process of the terminal reference point. In particular, this is also why the given initial state $x_{0}$ in (5) can be any real number.]

REMARK 2.2 Following the discussion of Remark 2.1, we see that our model models the situation where the investor concerns a reference wealth only at the terminal of the planning horizon (or, equivalently, an exogenously given dynamic reference trajectory). Examples of such a situation are when a person is to make a down payment of a house in three months (in which case the reference point is a deterministic constant), or when an investor is to cover the short position in a stock in one month (where the reference point is a random variable). 
It is certainly plausible that an investor will update his reference point dynamically. If the updating rule is known a priori, such as in Berkelaar et al. (2004), then it is possible to turn the problem into one covered by (6) by appropriately modifying some parameters. If, however, updating the reference point is part of the overall decision, then it would lead to a completely different and interesting model, which is open for further study.

REMARK 2.3 We implicitly assume in our model that the agent is a "small investor"; so his behavior only affects his utility function - and hence his asset allocation - but not the overall market. This is why the budget constraint in (7), $E[\rho X]=x_{0}$, is still evaluated in the conventional sense (no probability distortion). In other words, $E[\rho X]=x_{0}$ is the pricing rule of the market, which is (assumed to be) not influenced by the small investor under consideration.

Before we conclude this section, we recall the following definition. For any non-decreasing function $f: \mathbb{R}^{+} \mapsto \mathbb{R}^{+}$, we define its inverse function

$$
f^{-1}(x):=\inf \left\{y \in \mathbb{R}^{+}: f(y) \geq x\right\}, \quad x \in \mathbb{R}^{+} .
$$

It is immediate that $f^{-1}$ is non-decreasing and continuous on the left, and it holds always that

$$
f^{-1}(f(y)) \leq y
$$

\section{Ill-Posedness}

In general ill-posedness of an optimization problem signifies that the trade-off therein is not set right, leading to a wrong model. Well-posedness is an important issue from the modeling point of view. In classical portfolio selection literature [see, e.g., Karatzas and Shreve (1998)] the utility function is typically assumed to be globally concave along with other nice properties; thus the problem is guaranteed to be well-posed in most cases ${ }^{5}$. We now demonstrate that for the behavioral model (6) or (7) the well-posedness becomes a more significant issue, and that probability distortions in gains and losses play prominent, yet somewhat opposite, roles.

Theorem 3.1 Problem (7) is ill-posed if there exists a nonnegative $\mathcal{F}_{T}$-measurable random variable $X$ such that $E[\rho X]<+\infty$ and $V_{+}(X)=+\infty$.

Proof: Define $Y:=X-c$ with $c:=\left(E[\rho X]-x_{0}\right) / E \rho$. Then $Y$ is feasible for Problem (7). If $c \leq 0$, then obviously $V(Y)=V_{+}(Y) \geq V_{+}(X)=+\infty$. If $c>0$, then

$$
\begin{aligned}
V(Y) & =V_{+}\left(Y^{+}\right)-V_{-}\left(Y^{-}\right) \\
& \geq V_{+}\left(Y^{+}\right)-V_{-}(c) \\
& =\int_{0}^{+\infty} T_{+}\left(P\left\{u_{+}\left((X-c)^{+}\right)>y\right\}\right) d y-u_{-}(c)
\end{aligned}
$$

\footnotetext{
${ }^{5}$ Even with a global concave utility function the underlying problem could still be ill-posed; see counterexamples and discussions in Korn and Kraft (2004) and Jin, Xu and Zhou (2007).
} 


$$
\begin{aligned}
& =\int_{0}^{+\infty} T_{+}\left(P\left\{u_{+}(X-c)>y, X \geq c\right\}\right) d y-u_{-}(c) \\
& \geq \int_{0}^{+\infty} T_{+}\left(P\left\{u_{+}(X)>y+u_{+}(c)\right\}\right) d y-u_{-}(c) \\
& \geq \int_{u_{+}(c)}^{+\infty} T_{+}\left(P\left\{u_{+}(X)>y\right\}\right) d y-u_{-}(c) \\
& =+\infty
\end{aligned}
$$

where we have used the fact that $u_{+}(x+y) \leq u_{+}(x)+u_{+}(y) \forall x, y \in \mathbb{R}^{+}$due to the concavity of $u_{+}(\cdot)$ along with $u_{+}(0)=0$. The proof is complete.

Q.E.D.

This theorem says that the model is ill-posed if one can find a nonnegative claim having a finite price yet an infinite prospective value. In this case the agent can purchase such a claim initially (by taking out a loan if necessary) and reach the infinite value at the end. The following example shows that such an almost "unbelievable" claim could indeed exist even with very "nice" parameters involved, so long as the probability on gains is distorted.

EXAMPLE 3.1 Let $\rho$ be such that its (probability) distribution function, $F(\cdot)$, is continuous and strictly increasing, with $E \rho^{3}<+\infty$ (e.g., when $\rho$ is lognormal). Take $T_{+}(t):=t^{1 / 4}$ on $[0,1 / 2]$ and $u_{+}(x):=x^{1 / 2}$. Set $Z:=F(\rho)$. Then it is known that $Z \sim U(0,1)$, the uniform distribution on $(0,1)$. Define $X:=Z^{-1 / 2}-1$. Then $X \geq 0, P(X>x)=(1+x)^{-2}$ for $x \geq 0$, and

$$
E[\rho X]=E\left[\rho Z^{-1 / 2}\right]-E \rho \leq\left(E Z^{-3 / 4}\right)^{2 / 3}\left(E \rho^{3}\right)^{1 / 3}-E \rho=4^{2 / 3}\left(E \rho^{3}\right)^{1 / 3}-E \rho<+\infty .
$$

However,

$$
\begin{aligned}
V_{+}(X) & \geq \int_{2}^{+\infty} T_{+}\left(P\left\{X>y^{2}\right\}\right) d y=\int_{2}^{+\infty} T_{+}\left(\left(1+y^{2}\right)^{-2}\right) d y \\
& =\int_{2}^{+\infty}\left(1+y^{2}\right)^{-1 / 2} d y>\int_{2}^{+\infty}\left(2 y^{2}\right)^{-1 / 2} d y=+\infty
\end{aligned}
$$

In this example $u_{+}(x)=x^{1 / 2}$ is a perfectly "nice" utility function satisfying every condition required for well-posedness (as well as solvability) of the classical utility model; yet the distortion $T_{+}(\cdot)$ ruins everything and turns the problem into an ill-posed one.

To exclude the ill-posed case identified by Theorem 3.1, we need the following assumption throughout this paper:

Assumption $3.1 V_{+}(X)<+\infty$ for any nonnegative, $\mathcal{F}_{T}$-measurable random variable $X$ satisfying $E[\rho X]<+\infty$.

Assumption 3.1 is not sufficient to completely rule out the ill-posedness. The following theorem specifies another class of ill-posed problems.

Theorem 3.2 If $u_{+}(+\infty)=+\infty, \bar{\rho}=+\infty$, and $T_{-}(x)=x$, then Problem ( 7$)$ is ill-posed.

Proof: Fix any $a>\underline{\rho}$ and define $X:=c \mathbf{1}_{\rho<a}$ with $c:=\frac{x_{0}^{+}+1}{E\left[\rho \mathbf{1}_{\rho<a}\right]}>0$. Then for any $n>0$,

$$
\begin{aligned}
V_{+}(n X) & =\int_{0}^{+\infty} T_{+}\left(P\left\{u_{+}(n X)>y\right\}\right) d y \\
& =\int_{0}^{u_{+}(n c)} T_{+}\left(P\left\{u_{+}\left(n c \mathbf{1}_{\rho<a}\right)>y\right\}\right) d y \\
& =u_{+}(n c) T_{+}(P\{\rho<a\}) \rightarrow+\infty \text { as } n \rightarrow+\infty .
\end{aligned}
$$


Next, for any $n>1$, define $X_{n}:=c_{n} \mathbf{1}_{\rho>n^{2}}$, where $c_{n}:=\frac{n E[\rho X]-x_{0}}{E\left[\rho \mathbf{1}_{\rho>n^{2}}\right]}$. (Here $E\left[\rho \mathbf{1}_{\rho>n^{2}}\right]>0$ thanks to $\bar{\rho}=+\infty$.) Obviously, $c_{n}^{+} P\left\{\rho>n^{2}\right\}=\frac{\left(n E[\rho X]-x_{0}\right)^{+}}{E\left[\rho \mid \rho>n^{2}\right]} \leq \frac{\left|n E[\rho X]-x_{0}\right|}{n^{2}} \rightarrow 0$ as $n \rightarrow+\infty$. Hence

$$
V_{-}\left(c_{n}^{+} \mathbf{1}_{\rho>n^{2}}\right)=u_{-}\left(c_{n}^{+}\right) P\left\{\rho>n^{2}\right\} \leq u_{-}\left(c_{n}^{+} P\left\{\rho>n^{2}\right\}\right) \rightarrow 0 \text { as } n \rightarrow+\infty,
$$

where the last inequality is due to the facts that $u_{-}(\cdot)$ is concave and $u_{-}(0)=0$.

Now, define $\bar{X}_{n}:=n X-X_{n}$. Then $E\left[\rho \bar{X}_{n}\right]=n E[X \rho]-c_{n} E\left[\rho \mathbf{1}_{\rho>n^{2}}\right]=x_{0}$. Moreover, since $\bar{X}_{n}^{+} \geq n X$ and $\bar{X}_{n}^{-} \leq c_{n}^{+} \mathbf{1}_{\rho>n^{2}}$, it follows from (9) and (10) that $V\left(\bar{X}_{n}\right) \geq V_{+}(n X)-$ $V_{-}\left(c_{n}^{+} \mathbf{1}_{\rho>n^{2}}\right) \rightarrow+\infty$ as $n \rightarrow+\infty$.

Q.E.D.

REMARK 3.1 Quite intriguingly, Theorem 3.2 shows that a probability distortion on losses is necessary for the well-posedness if the utility on gains can go arbitrarily large (the latter being the case for most commonly used utility functions). The intuition behind this result and its proof can be explained as follows: one borrows enormous amount of money to purchase a claim with a huge payoff ( $n X$ in the proof), and then bet the market be "good" leading to the realization of that payoff. If, for the lack of luck, the market turns out to be "bad", then the agent ends up with a loss $\left(X_{n}\right)$; however due to the non-distortion on the loss side its damage on value is bounded [in fact equation (10) shows that the damage can be controlled to be arbitrarily small]. Notice that the above argument is no longer valid if the wealth is constrained to be bounded from below ${ }^{6}$.

Now we set out to identify and solve well-posed problems.

\section{Main Results}

The original problem (7) is solved in two steps involving three sub-problems, which are described in what follows.

Step 1. In this step we consider two problems respectively:

- Positive Part Problem: A problem with parameters $\left(A, x_{+}\right)$:

$$
\begin{array}{ll}
\text { Maximize } & V_{+}(X)=\int_{0}^{+\infty} T_{+}\left(P\left\{u_{+}(X)>y\right\}\right) d y \\
\text { subject to } & E[\rho X]=x_{+}, \quad X \geq 0 \text { a.s., } \quad X=0 \text { a.s. on } A^{C},
\end{array}
$$

where $x_{+} \geq x_{0}^{+}(\geq 0)$ and $A \in \mathcal{F}_{T}$ are given. Thanks to Assumption $3.1, V_{+}(X)$ is a finite number for any feasible $X$. We define the optimal value of Problem (11), denoted $v_{+}\left(A, x_{+}\right)$, in the following way. If $P(A)>0$, in which case the feasible region of (11) is non-empty $\left[X=\left(x_{+} \mathbf{1}_{A}\right) /(\rho P(A))\right.$ is a feasible solution], then $v_{+}\left(A, x_{+}\right)$is defined to be the supremum of (11). If $P(A)=0$ and $x_{+}=0$, then (11) has only one feasible solution $X=0$ a.s. and $v_{+}\left(A, x_{+}\right):=0$. If $P(A)=0$ and $x_{+}>0$, then (11) has no feasible solution, where we define $v_{+}(A, x):=-\infty$.

\footnotetext{
${ }^{6}$ This is why in Berkelaar et al. (2004) the model is well-posed even though no probability distortion is considered, as the wealth process there is constrained to be non-negative.
} 
- Negative Part Problem: A problem with parameters $\left(A, x_{+}\right)$:

$$
\begin{array}{ll}
\text { Minimize } & V_{-}(X)=\int_{0}^{+\infty} T_{-}\left(P\left\{u_{-}(X)>y\right\}\right) d y \\
\text { subject to } & \left\{\begin{array}{l}
E[\rho X]=x_{+}-x_{0}, X \geq 0 \text { a.s., } X=0 \text { a.s. on } A, \\
X \text { is upper bounded a.s., }
\end{array}\right.
\end{array}
$$

where $x_{+} \geq x_{0}^{+}$and $A \in \mathcal{F}_{T}$ are given. Similarly to the positive part problem we define the optimal value $v_{-}\left(A, x_{+}\right)$of Problem (12) as follows. When $P(A)<1$ in which case the feasible region of $(12)$ is non-empty, $v_{-}\left(A, x_{+}\right)$is the infimum of $(12)$. If $P(A)=1$ and $x_{+}=x_{0}$ where the only feasible solution is $X=0$ a.s., then $v_{-}\left(A, x_{+}\right):=0$. If $P(A)=1$ and $x_{+} \neq x_{0}$, then there is no feasible solution, in which case we define $v_{-}\left(A, x_{+}\right):=+\infty$.

Step 2. In this step we solve

$$
\begin{array}{ll}
\text { Maximize } & v_{+}\left(A, x_{+}\right)-v_{-}\left(A, x_{+}\right) \\
\text {subject to } & \left\{\begin{array}{l}
A \in \mathcal{F}_{T}, x_{+} \geq x_{0}^{+}, \\
x_{+}=0 \text { when } P(A)=0, x_{+}=x_{0} \text { when } P(A)=1 .
\end{array}\right.
\end{array}
$$

Let $F(\cdot)$ be the distribution function of $\rho$. Our main results are stated in terms of the following mathematical program, which is intimately related to (but not the same as) Problem (13):

$$
\begin{array}{ll}
\text { Maximize } & v_{+}\left(c, x_{+}\right)-u_{-}\left(\frac{x_{+}-x_{0}}{E\left[\rho \mathbf{1}_{\rho>c}\right]}\right) T_{-}(1-F(c)) \\
\text { subject to } & \left\{\begin{array}{c}
\underline{\rho} \leq c \leq \bar{\rho}, \quad x_{+} \geq x_{0}^{+}, \\
x_{+}=0 \text { when } c=\underline{\rho}, \quad x_{+}=x_{0} \text { when } c=\bar{\rho},
\end{array}\right.
\end{array}
$$

where $v_{+}\left(c, x_{+}\right):=v_{+}\left(\{\omega: \rho \leq c\}, x_{+}\right)$and we use the following convention:

$$
u_{-}\left(\frac{x_{+}-x_{0}}{E\left[\rho \mathbf{1}_{\rho>c}\right]}\right) T_{-}(1-F(c)):=0 \quad \text { when } c=\bar{\rho} \text { and } x_{+}=x_{0} .
$$

Here go the main results of this paper.

THEOREM 4.1 Assume that $u_{-}(\cdot)$ is strictly concave at 0 . We have the following conclusions:

(i) If $X^{*}$ is optimal for Problem (7), then $c^{*}:=F^{-1}\left(P\left\{X^{*} \geq 0\right\}\right)$, $x_{+}^{*}:=E\left[\rho\left(X^{*}\right)^{+}\right]$, where $F$ is the distribution function of $\rho$, are optimal for Problem (14). Moreover, $\left\{\omega: X^{*} \geq 0\right\}$ and $\left\{\omega: \rho \leq c^{*}\right\}$ are identical up to a zero probability set, and $\left(X^{*}\right)^{-}=$ $\frac{x_{+}^{*}-x_{0}}{E\left[\rho \mathbf{1}_{\rho>c^{*}}\right]} \mathbf{1}_{\rho>c^{*}}$ a.s..

(ii) If $\left(c^{*}, x_{+}^{*}\right)$ is optimal for Problem (14) and $X_{+}^{*}$ is optimal for Problem (11) with parameters $\left(\left\{\rho \leq c^{*}\right\}, x_{+}^{*}\right)$, then $X^{*}:=\left(X^{*}\right)^{+} \mathbf{1}_{\rho \leq c^{*}}-\frac{x_{+}^{*}-x_{0}}{E\left[\rho \mathbf{1}_{\rho>c^{*}}\right]} \mathbf{1}_{\rho>c^{*}}$ is optimal for Problem (7).

In the light of Theorem 4.1, we have the following algorithm to solve Problem (7). 
Step 1 Solve Problem (11) with $\left(\{\omega: \rho \leq c\}, x_{+}\right)$, where $\underline{\rho} \leq c \leq \bar{\rho}$ and $x_{+} \geq x_{0}^{+}$are given, to obtain $v_{+}\left(c, x_{+}\right)$and the optimal solution $X_{+}^{*}\left(c, x_{+}\right)$.

Step 2. Solve Problem (14) to get $\left(c^{*}, x_{+}^{*}\right)$.

Step 3. (i) If $\left(c^{*}, x_{+}^{*}\right)=\left(\bar{\rho}, x_{0}\right)$, then $X_{+}^{*}\left(\bar{\rho}, x_{0}\right)$ solves Problem (7).

(ii) Else $X_{+}^{*}\left(c^{*}, x_{+}^{*}\right) \mathbf{1}_{\rho \leq c^{*}}-\frac{x_{+}^{*}-x_{0}}{E\left[\rho \mathbf{1}_{\rho>c^{*}}\right]} \mathbf{1}_{\rho>c^{*}}$ solves Problem (7).

We now impose the following assumption:

Assumption $4.1 F^{-1}(z) / T_{+}^{\prime}(z)$ is non-decreasing in $z \in(0,1], \liminf _{x \rightarrow+\infty}\left(\frac{-x u_{+}^{\prime \prime}(x)}{u_{+}^{\prime}(x)}\right)>0$, and $E\left[u_{+}\left(\left(u_{+}^{\prime}\right)^{-1}\left(\frac{\rho}{T_{+}^{\prime}(F(\rho))}\right)\right) T_{+}^{\prime}(F(\rho))\right]<+\infty$.

Then $v_{+}\left(c, x_{+}\right)$and the corresponding optimal solution $X_{+}^{*}$ to (11) can be expressed more explicitly:

$$
\begin{aligned}
& v_{+}\left(c, x_{+}\right)=E\left[u_{+}\left(\left(u_{+}^{\prime}\right)^{-1}\left(\frac{\lambda\left(c, x_{+}\right) \rho}{T_{+}^{\prime}(F(\rho))}\right)\right) T_{+}^{\prime}(F(\rho)) \mathbf{1}_{\rho \leq c}\right], \\
& X_{+}^{*}=\left(u_{+}^{\prime}\right)^{-1}\left(\frac{\lambda\left(c, x_{+}\right) \rho}{T_{+}^{\prime}(F(\rho))}\right) \mathbf{1}_{\rho \leq c},
\end{aligned}
$$

where $\lambda\left(c, x_{+}\right)$satisfies $E\left[\left(u_{+}^{\prime}\right)^{-1}\left(\frac{\lambda\left(c, x_{+}\right) \rho}{T_{+}^{\prime}(F(\rho))}\right) \rho \mathbf{1}_{\rho \leq c}\right]=x_{+}$. In this case Theorem 4.1 can be re-stated with the preceding explicit expressions properly substituted.

Under Assumption 4.1, the optimal terminal wealth to our behavioral model (6) is given explicitly as the following

$$
X^{*}=\left(u_{+}^{\prime}\right)^{-1}\left(\frac{\lambda\left(c^{*}, x_{+}^{*}\right) \rho}{T_{+}^{\prime}(F(\rho))}\right) \mathbf{1}_{\rho \leq c^{*}}-\frac{x_{+}^{*}-x_{0}}{E\left[\rho \mathbf{1}_{\rho>c^{*}}\right]} \mathbf{1}_{\rho>c^{*}} .
$$

This solution possesses some appealing features. On one hand, the terminal wealth having a gain or a loss is completely determined by the terminal state density price being lower or higher than a single threshold, $c^{*}$, which in turn can be obtained by solving (14). On the other hand, (16) is the payoff of a combination of two binary options, which can be easily priced; see Appendix E.

The remainder of this paper is devoted to proving all the above claims. But before that, let us discuss on the economical interpretation of the optimal wealth profile (16). Indeed, (16) suggests that an optimal strategy should deliver a wealth in excess of the reference wealth in good states of the world $\left(\rho \leq c^{*}\right)$, and a shortfall in bad states $\left(\rho>c^{*}\right)^{7}$. To realize this goal, the agent should initially buy a contingent claim with the payoff $\left(u_{+}^{\prime}\right)^{-1}\left(\frac{\lambda\left(c^{*}, x_{+}^{*}\right) \rho}{T_{+}^{\prime}(F(\rho))}\right) \mathbf{1}_{\rho \leq c^{*}}$ at cost $x_{+}^{*}$. Since $x_{+}^{*} \geq x_{0}$, he needs to issue (i.e., sell) a claim with a payoff $\frac{x_{+}^{*}-x_{0}}{E\left[\rho 1_{\rho>c^{*}}\right]} \mathbf{1}_{\rho>c^{*}}$ to finance the shortfall, $x_{+}^{*}-x_{0}$. In other words, the agent will not only invest in stocks, but also will generally take a leverage to do so. He then gambles on a good state of the market turning up at the terminal time while accepting a fixed loss in case of a bad state ${ }^{8}$.

\footnotetext{
${ }^{7}$ It can be easily shown, in the case of a one-stock market, that $\rho \leq c^{*}$ is equivalent to the stock price exceeding a certain level.

${ }^{8}$ Such a gambling policy was derived in Berkelaar et al. (2004), Proposition 3, for a special model where the value function is a two-piece power function and there is no probability distortion. Here, we show that even for the most general model an optimal behavioral policy still possesses such an elegantly simple structure.
} 


\section{$5 \quad$ Splitting}

The key idea developed in this paper, i.e., splitting (7) into three sub-problems and then appropriately merging them, is based on the following observation: If $X$ is a feasible solution of (7), then one can split $X^{+}$and $X^{-}$. The former defines naturally an event $A:=\{X \geq 0\}$ and an initial price $x_{+}:=E\left[\rho X^{+}\right]$, and the latter corresponds to $A^{C}$ and $x_{+}-x_{0}$, where $A^{C}$ denotes the complement of the set $A$. An optimal solution to (7) should, therefore, induce the "best" such $A$ and $x_{+}$in certain sense. We now prove that this idea indeed works in the sense that (7) is equivalent to the three auxiliary problems combined.

We start with the well-posedness.

Proposition 5.1 Problem (7) is ill-posed if and only if Problem (13) is ill-posed.

Proof: We first show the "if" part. Suppose (13) is ill-posed. If $v_{+}\left(\Omega, x_{0}\right)=+\infty$, then Problem (7) is obviously ill-posed. If $v_{+}\left(\Omega, x_{0}\right)<+\infty$, then for any $M>v_{+}\left(\Omega, x_{0}\right)$, there exists a feasible pair $\left(A, x_{+}\right)$for $(13)$ such that $v_{+}\left(A, x_{+}\right)-v_{-}\left(A, x_{+}\right) \geq M$. Clearly $0<$ $P(A)<1$. (If $P(A)=0$, then $v_{+}\left(A, x_{+}\right)-v_{-}\left(A, x_{+}\right) \leq 0<M$. If $P(A)=1$, then $v_{+}\left(A, x_{+}\right)-v_{-}\left(A, x_{+}\right) \leq v_{+}\left(\Omega, x_{0}\right)<M$. $)$ Consequently, both (11) and (12) with parameters $\left(A, x_{+}\right), x_{+} \geq x_{0}^{+}$, have non-empty feasible regions. So there exist $X_{1}$ and $X_{2}$ feasible for (11) and (12) respectively such that $V_{+}\left(X_{1}\right) \geq v_{+}\left(A, x_{+}\right)-1, V_{-}\left(X_{2}\right) \leq v_{-}\left(A, x_{+}\right)+1$. Define $X=X_{1}-X_{2}$. Then $X$ is feasible for (7), and $V(X) \geq v_{+}\left(A, x_{+}\right)-v_{-}\left(A, x_{+}\right)-2 \geq M-2$, implying that (13) is ill-posed.

For the "only if" part, if (7) is ill-posed, then for any $M>0$, there exists a feasible solution $X$ for $(7)$ such that $V(X) \geq M$. Define $A:=\{\omega: X \geq 0\}, x_{+}:=E\left[\rho X^{+}\right]$. Then $\left(A, x_{+}\right)$ is feasible for Problem (13), and $v_{+}\left(A, x_{+}\right)-v_{-}\left(A, x_{+}\right) \geq V\left(X^{+}\right)-V\left(X_{-}\right)=V(X) \geq M$, which shows that Problem (13) is ill-posed.

Q.E.D.

Proposition 5.2 Given $X^{*}$, define $A^{*}:=\left\{\omega: X^{*} \geq 0\right\}$ and $x_{+}^{*}:=E\left[\rho\left(X^{*}\right)^{+}\right]$. Then $X^{*}$ is optimal for Problem ( 7$)$ if and only if $\left(A^{*}, x_{+}^{*}\right)$ are optimal for Problem (13) and $\left(X^{*}\right)^{+}$and $\left(X^{*}\right)^{-}$are respectively optimal for Problems (11) and (12) with parameters $\left(A^{*}, x_{+}^{*}\right)$.

Proof: For the "if" part, we first have $V\left(X^{*}\right)=v_{+}\left(A^{*}, x_{+}^{*}\right)-v_{-}\left(A^{*}, x_{+}^{*}\right)$. For any feasible solution $X$ of $(7)$, define $A:=\{\omega: X \geq 0\}$ and $x_{+}:=E\left[\rho X^{+}\right]$. Then we have $V_{+}\left(X^{+}\right) \leq v_{+}\left(A, x_{+}\right), V_{-}\left(X^{-}\right) \geq v_{-}\left(A, x_{+}\right)$. Therefore $V(X)=V_{+}\left(X^{+}\right)-V_{-}\left(X^{-}\right) \leq$ $v_{+}\left(A, x_{+}\right)-v_{-}\left(A, x_{+}\right) \leq v_{+}\left(A^{*}, x_{+}^{*}\right)-v_{-}\left(A^{*}, x_{+}^{*}\right)=V\left(X^{*}\right)$, which means $X^{*}$ is optimal for $(7)$.

For the "only if" part, let $X^{*}$ be optimal for (7). Obviously, $V_{+}\left(\left(X^{*}\right)^{+}\right) \leq v_{+}\left(A^{*}, x_{+}^{*}\right)$ and $V_{-}\left(\left(X^{*}\right)^{-}\right) \geq v_{-}\left(A^{*}, x_{+}^{*}\right)$. If the former holds strictly, then there exists $X_{1}$ feasible for (11) with parameters $\left(A^{*}, x_{+}^{*}\right)$ such that $V_{+}\left(X_{1}\right)>V_{+}\left(\left(X^{*}\right)^{+}\right)$. As a result $\bar{X}:=X_{1} \mathbf{1}_{A^{*}}+X^{*} \mathbf{1}_{\left(A^{*}\right)^{C}}$ is feasible for $(7)$ and $V(\bar{X})>V\left(X^{*}\right)$, which contradicts the optimality of $X^{*}$. So $\left(X^{*}\right)^{+}$is optimal for (11). Similarly we can prove that $\left(X^{*}\right)^{-}$is optimal for (12). Thus $v_{+}\left(A^{*}, x_{+}^{*}\right)=$ $V_{+}\left(\left(X^{*}\right)^{+}\right), v_{-}\left(A^{*}, x_{+}^{*}\right)=V_{-}\left(\left(X^{*}\right)^{-}\right)$.

Next we show that $v_{+}\left(A, x_{+}\right)-v_{-}\left(A, x_{+}\right) \leq v_{+}\left(A^{*}, x_{+}^{*}\right)-v_{-}\left(A^{*}, x_{+}^{*}\right) \equiv V\left(X^{*}\right)$ for any feasible pair $\left(A, x_{+}\right)$of Problem (13). This can be proved in three cases: 
(i) If $P(A)=0$ (hence $x_{+}=0$ and $x_{0} \leq 0$ ), then

$$
\begin{aligned}
v_{+}\left(A, x_{+}\right)-v_{-}\left(A, x_{+}\right) & =-v_{-}(A, 0) \\
& =-v_{-}\left(A, x_{0}^{+}\right) \\
& =E[\rho X]=x_{0}^{-}, X \geq 0, X \text { is upper bounded }\left[-V_{-}(X)\right] \\
& =E(X) \\
& \leq \quad \text { sup } \sup _{[\rho X]=-x_{0}^{-}, X \leq 0, X \text { is lower bounded }} V(X) \\
& =V\left(X^{*}\right),
\end{aligned}
$$

where the last equality is owing to the fact that $-x_{0}^{-}=x_{0}$.

(ii) If $P(A)=1$ (hence $\left.x_{+}=x_{0}\right)$, then we need only to check $v_{+}\left(A, x_{0}\right) \leq V\left(X^{*}\right)$, which is easy since $v_{+}\left(A, x_{0}\right)=\sup _{E[\rho X]=x_{0}, X \geq 0} V(X)$.

(iii) If $0<P(A)<1$, then for any $x_{+} \geq x_{0}^{+}$, both (11) and (12) with parameters $\left(A, x_{+}\right)$ have non-empty feasible regions. Hence for any $\epsilon>0$ there exist $X_{1}$ and $X_{2}$, feasible for (11) and (12) respectively, such that $V_{+}\left(X_{1}\right)>\left(v_{+}\left(A, x_{+}\right)-\epsilon\right), V_{-}\left(X_{2}\right)<v_{-}\left(A, x_{+}\right)+\epsilon$. Letting $X:=X_{1}-X_{2}$, which is feasible for $(7)$, we have $v_{+}\left(A, x_{+}\right)-v_{-}\left(A, x_{+}\right)<$ $V_{+}\left(X_{1}\right)-V_{-}\left(X_{2}\right)+2 \epsilon=V(X)+2 \epsilon \leq V\left(X^{*}\right)+2 \epsilon$.

This concludes the proof.

Q.E.D.

The essential message of Propositions 5.1 and 5.2 is that our problem (7) is completely equivalent to the set of problems (11) - (13) and, moreover, the solution to the former can be obtained via those to the latter.

Problem (13) is an optimization problem with the decision variables being a real number, $x_{+}$, and a random event, $A$, the latter being very hard to handle. We now show that one needs only to consider $A=\{\rho \leq c\}$, where $c$ is a real number in certain range, when optimizing (13).

Recall that two random variables $\xi$ and $\eta$ are called comonotonic (anti-comonotonic respectively) if $\left[\xi(\omega)-\xi\left(\omega^{\prime}\right)\right]\left[\eta(\omega)-\eta\left(\omega^{\prime}\right)\right] \geq(\leq$ respectively $) \quad 0$.

TheOREm 5.1 For any feasible pair $\left(A, x_{+}\right)$of Problem (13), there exists $c \in[\rho, \bar{\rho}]$ such that $\bar{A}:=\{\omega: \rho \leq c\}$ satisfies

$$
v_{+}\left(\bar{A}, x_{+}\right)-v_{-}\left(\bar{A}, x_{+}\right) \geq v_{+}\left(A, x_{+}\right)-v_{-}\left(A, x_{+}\right) .
$$

Moreover, if Problem (11) admits an optimal solution with parameters $\left(A, x_{+}\right)$, then the inequality in (17) is strict unless $P\left(A \cap \bar{A}^{C}\right)+P\left(A^{C} \cap \bar{A}\right)=0$.

Proof: The case when $x_{+}=x_{0}^{+}$is trivial. In fact, if $x_{0} \leq 0$, then $x_{+}=0$ and $v_{+}\left(A, x_{+}\right)=$ $0 \forall A$; hence $c=\underline{\rho}$ or $\bar{A}=\varnothing$. If $x_{0}>0$, then obviously $v_{-}\left(A, x_{+}\right)=0 \forall A$; hence (17) holds with $\bar{A}=\Omega$ or $c=\bar{\rho}$. On the other hand, the case when $P(A)=0$ or $P(A)=1$ is also trivial, where $c:=\underline{\rho}$ or $c:=\bar{\rho}$ trivially meets $(17)$. 
So we assume now that $x_{+}>x_{0}^{+}$and $0<P(A)<1$. Denote $\alpha:=P(A), B:=A^{C}$. Let $\bar{A}=\{\omega: \rho \leq c\}$, where $c \in[\underline{\rho}, \bar{\rho})$ satisfies $P\{\rho \leq c\}=\alpha$. Further, set

$$
\begin{array}{ll}
A_{1}=A \cap\{\omega: \rho \leq c\}, & A_{2}=A \cap\{\omega: \rho>c\}, \\
B_{1}=B \cap\{\omega: \rho \leq c\}, & B_{2}=B \cap\{\omega: \rho>c\} .
\end{array}
$$

Since $P\left(A_{1} \cup B_{1}\right)=P\left(A_{1} \cup A_{2}\right) \equiv \alpha$, we conclude $P\left(A_{2}\right)=P\left(B_{1}\right)$.

If $P\left(A_{2}\right)=P\left(B_{1}\right)=0$, then trivially $v_{+}\left(\bar{A}, x_{+}\right)-v_{-}\left(\bar{A}, x_{+}\right)=v_{+}\left(A, x_{+}\right)-v_{-}\left(A, x_{+}\right)$. So we suppose $P\left(A_{2}\right)=P\left(B_{1}\right)>0$. For any feasible solutions $X_{1}$ and $X_{2}$ for (11) and (12), respectively, with parameters $\left(A, x_{+}\right)$, we are to prove that

$$
V_{+}\left(X_{1}\right)-V_{-}\left(X_{2}\right) \leq v_{+}\left(\bar{A}, x_{+}\right)-v_{-}\left(\bar{A}, x_{+}\right)
$$

To this end, define $f_{1}(t):=P\left\{X_{1} \leq t \mid A_{2}\right\}, g_{1}(t):=P\left\{\rho \leq t \mid B_{1}\right\}, t \geq 0, Z_{1}:=g_{1}(\rho)$ and $Y_{1}:=f_{1}^{-1}\left(Z_{1}\right)$. Because $\rho$ admits no atom with respect to $P$, it admits no atom with respect to $P\left(\cdot \mid B_{1}\right)$. Hence the distribution of $Z_{1}$ conditional on $B_{1}$ is $U(0,1)$, which implies $P\left\{Y_{1} \leq t \mid B_{1}\right\}=P\left\{Z_{1} \leq f_{1}(t) \mid B_{1}\right\}=f_{1}(t)$. Consequently,

$$
\begin{aligned}
E\left[\rho X_{1} \mathbf{1}_{A_{2}}\right] \geq c E\left[X_{1} \mathbf{1}_{A_{2}}\right] & =c P\left(A_{2}\right) E\left[X_{1} \mid A_{2}\right] \\
& =c P\left(B_{1}\right) \int_{0}^{+\infty}\left[1-f_{1}(t)\right] d t \\
& =c P\left(B_{1}\right) \int_{0}^{+\infty} P\left\{Y_{1}>t \mid B_{1}\right\} d t \\
& =c E\left[Y_{1} \mathbf{1}_{B_{1}}\right] \\
& \geq E\left[\rho Y_{1} \mathbf{1}_{B_{1}}\right]
\end{aligned}
$$

and the inequality is strict if and only if $P\left\{X_{1}>0\right\}>0$ or $f_{1}(t) \not \equiv 1$.

Define

$$
k_{1}:= \begin{cases}1, & \text { if } Y_{1}=0, \text { a.s. on } B_{1}, \\ \frac{E\left[\rho X_{1} \mathbf{1}_{A_{2}}\right]}{E\left[\rho Y_{1} \mathbf{1}_{B_{1}}\right]}, & \text { otherwise. }\end{cases}
$$

Then $k_{1} \geq 1$, and $k_{1}>1$ if and only if $f_{1}(t) \not \equiv 1$. Set $\bar{X}_{1}:=X_{1} \mathbf{1}_{A_{1}}+k_{1} Y_{1} \mathbf{1}_{B_{1}}$. Then

$$
E\left[\rho X_{1}\right]=E\left[\rho X_{1} \mathbf{1}_{A_{1}}\right]+E\left[\rho X_{1} \mathbf{1}_{A_{2}}\right]=E\left[\rho X_{1} \mathbf{1}_{A_{1}}\right]+E\left[k_{1} \rho Y_{1} \mathbf{1}_{B_{1}}\right]=E\left[\rho \bar{X}_{1}\right],
$$

which means that $\bar{X}_{1}$ is feasible for $(11)$ with parameters $\left(\bar{A}, x_{+}\right)$(recall that by definition $\bar{X}_{1}=0$ on $\bar{A}^{C}$ ).

On the other hand, for any $t>0$,

$$
\begin{aligned}
P\left\{\bar{X}_{1}>t\right\} & =P\left\{\bar{X}_{1}>t \mid A_{1}\right\} P\left(A_{1}\right)+P\left\{\bar{X}_{1}>t \mid B_{1}\right\} P\left(B_{1}\right) \\
& =P\left\{X_{1}>t \mid A_{1}\right\} P\left(A_{1}\right)+P\left\{k_{1} Y_{1}>t \mid B_{1}\right\} P\left(B_{1}\right) \\
& \geq P\left\{X_{1}>t \mid A_{1}\right\} P\left(A_{1}\right)+P\left\{Y_{1}>t \mid B_{1}\right\} P\left(B_{1}\right) \\
& =P\left\{X_{1}>t \mid A_{1}\right\} P\left(A_{1}\right)+P\left\{X_{1}>t \mid A_{2}\right\} P\left(A_{2}\right) \\
& =P\left\{X_{1}>t\right\},
\end{aligned}
$$


and the equality holds for a.e. $t \geq 0$ if and only if $f_{1}(t) \equiv 1$.

It follows then by the definition of $V_{+}(\cdot)$ that

$$
V_{+}\left(\bar{X}_{1}\right) \geq V_{+}\left(X_{1}\right)
$$

with the inequality being strict when $f_{1}(\cdot) \not \equiv 1$.

In a similar fashion we can construct $\bar{X}_{2}$ feasible for $(12)$ with parameters $\left(\bar{A}, x_{+}\right)$satisfying

$$
V_{-}\left(\bar{X}_{2}\right) \leq V_{-}\left(X_{2}\right)
$$

Combining (19) and (20) we get (18).

Now, if $X_{1}$ is an optimal solution of (11) with parameters $\left(A, x_{+}\right)$, then $P\left(X_{1}=0 \mid A_{2}\right)<1$. Indeed, if $P\left(X_{1}=0 \mid A_{2}\right)=1$, then by its optimality $X_{1}$ is anti-comonotonic with $\rho$ on $A$ (see Proposition C.1), which implies $P\left(X_{1}=0 \mid A_{1}\right)=1$. Therefore $P\left(X_{1}=0 \mid A\right)=1$, and $x_{+}=E\left[\rho X_{1} \mathbf{1}_{A}\right]=0$, contradicting the fact that $x_{+}>x_{0}^{+} \geq 0$.

Thus, $f_{1}(\cdot) \not \equiv 1$. As proved earlier, (19), and hence (18), holds strictly. Q.E.D.

To simplify the notation, we now use $v_{+}\left(c, x_{+}\right)$and $v_{-}\left(c, x_{+}\right)$to denote $v_{+}\left(\{\omega: \rho \leq c\}, x_{+}\right)$ and $v_{-}\left(\{\omega: \rho \leq c\}, x_{+}\right)$respectively.

In view of Theorem 5.1, one may replace Problem (13) by the following problem:

$$
\begin{array}{ll}
\text { Maximize } & v_{+}\left(c, x_{+}\right)-v_{-}\left(c, x_{+}\right) \\
\text {subject to } & \left\{\begin{array}{l}
\underline{\rho} \leq c \leq \bar{\rho}, \quad x_{+} \geq x_{0}^{+}, \\
x_{+}=0 \text { when } c=\underline{\rho}, x_{+}=x_{0} \text { when } c=\bar{\rho} .
\end{array}\right.
\end{array}
$$

This is clearly a much simpler problem, being a constrained optimization problem (a mathematical programming problem) in $\mathbb{R}^{2}$.

Theorem 5.1 is one of the most important results in this paper. It discloses the form of a general solution to the behavioral model: the optimal wealth is the payoff of a combination of two binary options characterized by a single number $c^{*}$, as stipulated in the next theorem.

Theorem 5.2 Given $X^{*}$, and define $c^{*}:=F^{-1}\left(P\left\{X^{*} \geq 0\right\}\right), x_{+}^{*}:=E\left[\rho\left(X^{*}\right)^{+}\right]$, where $F(\cdot)$ is the distribution function of $\rho$. Then $X^{*}$ is optimal for Problem ( 7$)$ if and only if $\left(c^{*}, x_{+}^{*}\right)$ is optimal for Problem (21) and $\left(X^{*}\right)^{+} \mathbf{1}_{\rho \leq c^{*}}$ and $\left(X^{*}\right)^{-} \mathbf{1}_{\rho>c^{*}}$ are respectively optimal for Problems (11) and (12) with parameters $\left(\left\{\omega: \rho \leq c^{*}\right\}, x_{+}^{*}\right)$. Moreover, in this case $\left\{\omega: X^{*} \geq 0\right\}$ and $\left\{\omega: \rho \leq c^{*}\right\}$ are identical up to a zero probability set.

PROOF: Straightforward from Proposition 5.2 and Theorem 5.1.

Q.E.D.

In the following two sections, we will solve the positive and negative part problems respectively to obtain $v_{+}\left(c, x_{+}\right)$and $v_{-}\left(c, x_{+}\right)$. It turns out that the two problems require very different techniques to tackle. 


\section{Positive Part Problem}

In this section we solve the positive part problem (11), including finding its optimal solution and the expression of $v_{+}\left(c, x_{+}\right)$, for any $A=\{\omega: \rho \leq c\}, \underline{\rho} \leq c \leq \bar{\rho}$, and $x_{+} \geq x_{0}^{+}$. In fact, it is a special case of a more general Choquet maximization problem, which is of independent interest and is solved in Appendix C.

\subsection{Solving (11)}

We apply the general results obtained in Appendix $\mathrm{C}$ to Problem (11) with $A=\{\omega: \rho \leq c\}$ and $x_{+} \geq x_{0}^{+}(\geq 0)$. Let $F(\cdot)$ be the distribution function of $\rho$.

Let $A=\{\omega: \rho \leq c\}$ be given. Problem (11) is trivial when $P(A)=0$; hence we assume $P(A)>0$ or $c>\underline{\rho}$. Define

$$
T_{A}(x):=T_{+}(x P(A)) / T_{+}(P(A)), \quad x \in[0,1],
$$

which is a strictly increasing, differentiable function from $[0,1]$ to $[0,1]$, with $T_{A}(0)=0, T_{A}(1)=$ 1. For any feasible solution $X$ of (11) and any $y \geq 0$,

$$
T_{+}\left(P\left\{u_{+}(X)>y\right\}\right)=T_{+}\left(P\left\{u_{+}(X)>y \mid A\right\} P(A)\right)=T_{+}(P(A)) T_{A}\left(P\left\{u_{+}(X)>y \mid A\right\}\right) .
$$

Now considering Problem (11) in the conditional probability space $\left(\Omega \cap A, \mathcal{F} \cap A, P_{A}:=P(\cdot \mid A)\right)$, we can rewrite it as

$$
\begin{array}{ll}
\text { Maximize } & V_{+}(Y)=T_{+}(P(A)) \int_{0}^{+\infty} T_{A}\left(P_{A}\left\{u_{+}(Y)>y\right\}\right) d y \\
\text { subject to } & E_{A}[\rho Y]=x_{+} / P(A), \quad Y \geq 0 .
\end{array}
$$

This specializes the general Choquet maximization problem (46) solved in Appendix C. It is evident that $Y^{*}$ is optimal for (22) if and only if $X^{*}=Y^{*} \mathbf{1}_{A}$ is optimal for (11).

To solve Problem (11) for all $A=\{\omega: \rho \leq c\}$, we need Assumption 4.1.

Theorem 6.1 Let Assumption 4.1 hold. Given $A:=\{\omega: \rho \leq c\}$ with $\underline{\rho} \leq c \leq \bar{\rho}$, and $x_{+} \geq x_{0}^{+}$.

(i) If $x_{+}=0$, then the optimal solution of (11) is $X^{*}=0$ and $v_{+}\left(c, x_{+}\right)=0$.

(ii) If $x_{+}>0$ and $c=\underline{\rho}$, then there is no feasible solution to (11) and $v_{+}\left(c, x_{+}\right)=-\infty$.

(iii) If $x_{+}>0$ and $\underline{\rho}<c \leq \bar{\rho}$, then the optimal solution to (11) is $X^{*}(\lambda)=\left(u_{+}^{\prime}\right)^{-1}\left(\frac{\lambda \rho}{T_{+}^{\prime}(F(\rho))}\right) \mathbf{1}_{\rho \leq c}$ with the optimal value $v_{+}\left(c, x_{+}\right)=E\left[u_{+}\left(\left(u_{+}^{\prime}\right)^{-1}\left(\frac{\lambda \rho}{T_{+}^{\prime}(F(\rho))}\right)\right) T_{+}^{\prime}(F(\rho)) \mathbf{1}_{\rho \leq c}\right]$, where $\lambda>$ 0 is the unique real number satisfying $E\left[\rho X^{*}(\lambda)\right]=x_{+}$.

Proof: Cases (i) and (ii) are trivial. We prove (iii). Assume $\underline{\rho}<c \leq \bar{\rho}$ with $P(A) \equiv$ $P\{\rho \leq c\}>0$. Define $F_{A}(x):=P_{A}\{\rho \leq x\}=\frac{P\{\rho \leq x \wedge c\}}{P\{\rho \leq c\}}=\frac{F(x \wedge c)}{P(A)}, x \geq 0$. Then $F_{A}^{-1}(x)=$ $F^{-1}(x P(A))$. Noting $T_{A}^{\prime}(x)=\frac{P(A)}{T_{+}(P(A))} T_{+}^{\prime}(x P(A))$, we have $\frac{F_{A}^{-1}(z)}{T_{A}^{\prime}(z)}=\frac{F^{-1}(z P(A))}{T_{+}^{\prime}(z P(A))} \frac{T_{+}(P(A))}{P(A)}$, which 
is non-decreasing in $z$ under Assumption 4.1. Noting that $\rho \leq c$ on $A$, we have $\frac{\rho}{T_{A}^{\prime}\left(F_{A}(\rho)\right)}=$ $\frac{\rho}{T_{+}^{\prime}(F(\rho))} \frac{T_{+}(P(A))}{P(A)}$. Hence, in view of Assumption 4.1 and Proposition C.2 we can apply Theorem C.1 to conclude that the optimal solution for $(22)$ is $Y^{*}=\left(u_{+}^{\prime}\right)^{-1}\left(\frac{\bar{\lambda} \rho}{T_{A}^{\prime}\left(F_{A}(\rho)\right)}\right)$ for some $\bar{\lambda}>0$. Denoting $\lambda:=\frac{T_{+}(P(A))}{P(A)} \bar{\lambda} \geq 0$, we obtain the optimality of $X^{*}:=Y^{*} \mathbf{1}_{\rho \leq c}$ in view of the relation between Problems (22) and (11).

Finally, the optimal value of Problem (11) can be calculated as follows:

$$
\begin{aligned}
v_{+}\left(c, x_{+}\right) & =T_{+}(P(A)) E_{A}\left[u_{+}\left(Y^{*}\right) T_{A}^{\prime}\left(F_{A}(\rho)\right)\right] \\
& =P(A) E_{A}\left[u_{+}\left(Y^{*}\right) T_{+}^{\prime}(F(\rho))\right] \\
& =E\left[u_{+}\left(Y^{*}\right) T_{+}^{\prime}(F(\rho)) \mathbf{1}_{\rho \leq c}\right] .
\end{aligned}
$$

The proof is complete.

Q.E.D.

Theorem 6.1 remains true when the condition $\liminf \inf _{x \rightarrow+\infty}\left(\frac{-x u_{+}^{\prime \prime}(x)}{u_{+}^{\prime}(x)}\right)>0$ in Assumption 4.1 is replaced by a (mathematically) weaker one

$$
\limsup _{x \rightarrow+\infty} \frac{u_{+}^{\prime}(k x)}{u_{+}^{\prime}(x)}<1 \text { for some } k>1,
$$

which, in particular, does not require the twice differentiability of $u_{+}(\cdot)$; see Jin, Xu and Zhou (2007, Lemma 3 and Proposition 2). We choose to use the current condition due to its clear economic meaning related to the relative risk aversion index.

Before we end this subsection, we state the following result which is useful in the sequel.

Proposition 6.1 If $x_{+}>0$, then Problem (11) admits an optimal solution with parameters $\left(\{\rho \leq c\}, x_{+}\right)$only if $v_{+}\left(\bar{c}, x_{+}\right)>v_{+}\left(c, x_{+}\right)$for any $\bar{c}>c$ satisfying $P\{c<\rho \leq \bar{c}\}>0$.

Proof: Assume $c>\underline{\rho}$, the case $c=\underline{\rho}$ being trivial. Let $X$ be optimal for (11) with $\left(A(c), x_{+}\right)$, where $A(c):=\{\omega: \rho \leq c\}$. Then $Y_{c}:=\left.X\right|_{A(c)}$, where $\left.X\right|_{A(c)}$ is $X$ restricted on $A(c)$, is optimal for (22) with $A=A(c)$.

For any $\bar{c}>c$, obviously $v_{+}\left(\bar{c}, x_{+}\right) \geq v_{+}\left(c, x_{+}\right)$. If $v_{+}\left(\bar{c}, x_{+}\right)=v_{+}\left(c, x_{+}\right)$, then, with $A(\bar{c}):=\{\omega: \rho \leq \bar{c}\}$, the random variable

$$
\bar{Y}(\omega):= \begin{cases}Y_{c}(\omega), & \text { if } \omega \in A(c), \\ 0, & \text { if } \omega \in A(\bar{c}) \backslash A(c)\end{cases}
$$

is feasible for $(22)$ with $A=A(\bar{c})$ and, since its objective value is $v_{+}\left(c, x_{+}\right)=v_{+}\left(\bar{c}, x_{+}\right)$, is optimal. By Theorem C.2, $P\{\bar{Y}=0 \mid A(\bar{c})\}=0$. However, the definition of $\bar{Y}$ shows that $P\{\bar{Y}=0 \mid A(\bar{c})\}>0$ if $P\{c<\rho \leq \bar{c}\}>0$. This contradiction leads to $v_{+}\left(\bar{c}, x_{+}\right)>v_{+}\left(c, x_{+}\right)$. Q.E.D.

In other words, $v_{+}$is strictly increasing in $c$. 


\subsection{Discussion on the Monotonicity of $F^{-1}(z) / T_{+}^{\prime}(z)$}

It is seen from the previous subsections that in order to solve the positive part problem explicitly, a key assumption is the monotonicity of $F^{-1}(z) / T_{+}^{\prime}(z)$. What is the economic interpretation of this property? Does it contradict the other assumptions usually imposed on $F(\cdot)$ and $T_{+}(\cdot)$ ? More importantly, is the set of the problem parameters satisfying this assumption null in the first place? In this subsection we depart from our optimization problems for a while to address these questions ${ }^{9}$.

Throughout this subsection, we assume that $F(\cdot)$ (the distribution function of $\rho$ ) is twice differentiable and $F^{\prime}(x)>0 \forall x>0$ (e.g., when $\rho$ is a non-degenerate lognormal random variable). Furthermore, suppose that $T_{+}(\cdot)$ is twice differentiable on $(0,1)$.

Denote $x=F^{-1}(z)$ or $z=F(x)$. Then the monotonicity (being non-decreasing) of $F^{-1}(z) / T_{+}^{\prime}(z)$ is equivalent to that $T_{+}^{\prime}(F(x)) / x$ is non-increasing in $x>0$. Set $H(x):=$ $T_{+}(F(x)), h(x):=H^{\prime}(x)$, and $I(x):=T_{+}^{\prime}(F(x)) / x \equiv h(x) /\left(x F^{\prime}(x)\right), x>0$. Then $I(x)$ non-increases in $x>0$ if and only if

$$
I^{\prime}(x)=\frac{x H^{\prime \prime}(x) F^{\prime}(x)-x H^{\prime}(x) F^{\prime \prime}(x)-H^{\prime}(x) F^{\prime}(x)}{x^{2}\left(F^{\prime}(x)\right)^{2}} \leq 0 \quad \forall x>0,
$$

which is further equivalent to

$$
\frac{x H^{\prime \prime}(x)}{H^{\prime}(x)}-\frac{x F^{\prime \prime}(x)}{F^{\prime}(x)} \leq 1 \quad \forall x>0
$$

or

$$
\left(\ln H^{\prime}(x)\right)^{\prime} \leq\left(\ln \left(x F^{\prime}(x)\right)\right)^{\prime} \forall x>0
$$

Note that $\frac{x u^{\prime \prime}(x)}{u^{\prime}(x)}$ can be regarded as the relative risk seeking index of a given function $u(\cdot)$. On the other hand, recall that by definition $H(\cdot)$ is the distorted distribution function of $\rho$. Hence the condition (23) can be economically interpreted as that the distortion $T_{+}$should not be "too large" in the sense that it should not increase the relative risk seeking function of the distribution by more than 1 .

Next we are to explore more properties of the function $j(\cdot)$ defined by $j(x):=\frac{x H^{\prime \prime}(x)}{H^{\prime}(x)}-$ $\frac{x F^{\prime \prime}(x)}{F^{\prime}(x)}, \quad x>0$. To this end, let $G(z):=F^{-1}(z)$. Then $G^{\prime}(z)=\frac{1}{F^{\prime}(G(z))} \forall z \in(0,1)$. Since $T_{+}(z)=H(G(z))$, we have $T_{+}^{\prime}(z)=h(G(z)) / F^{\prime}(G(z))$; hence

$$
T_{+}^{\prime \prime}(z)=\frac{h^{\prime}(G(z)) F^{\prime}(G(z))-h(G(z)) F^{\prime \prime}(G(z))}{F^{\prime}(G(z))^{3}} .
$$

This leads to

$$
T_{+}^{\prime \prime}(F(x))=\frac{h^{\prime}(x) F^{\prime}(x)-h(x) F^{\prime \prime}(x)}{F^{\prime}(x)^{3}}=\frac{h(x)}{x F^{\prime}(x)^{2}} j(x), x>0 .
$$

As proposed by Tversky and Kahneman (1992), the probability distortion $T_{+}(\cdot)$ is usually in reversed S-shape. Specifically, $T_{+}(x)$ changes from being concave to being convex when $x$ goes from 0 to 1 , or $T_{+}^{\prime \prime}(x)$ changes from negative to positive. It follows then from (25) that

\footnotetext{
${ }^{9}$ The reader may skip this subsection without interrupting the flow of reading.
} 
$j(\cdot)$ changes from negative to positive when $x$ goes from 0 to 1 , while as shown earlier $(23)$ requires that $j(\cdot)$ is bounded above by 1 .

To summarize, a reversed S-shaped distortion $T_{+}(\cdot)$ satisfying the monotonicity condition in Assumption 4.1 if there exists $c_{0}>0$ such that

$$
j(x) \leq 0 \quad \forall x \in\left(0, c_{0}\right], \text { and } 0 \leq j(x) \leq 1 \quad \forall x \in\left(c_{0},+\infty\right) .
$$

The following is an example of distortion where the corresponding $j(\cdot)$ does satisfy $(26)$.

EXAMPLE 6.1 Let $\rho$ be a non-degenerate lognormal random variable; i.e., $F(x)=N\left(\frac{\ln x-\mu}{\sigma}\right)$ for some $\mu \in \mathbb{R}$ and $\sigma>0$, where $N(\cdot)$ is the distribution function of a standard normal random variable. Take $j(x)=: a \mathbf{1}_{0<x \leq c_{0}}+b \mathbf{1}_{x>c_{0}}$, with $c_{0}>0, a<0$ and $0<b<1$ all given. This is the "simplest" function satisfying (26). We now track down the distortion $T_{+}(\cdot)$ that produces the function $j(\cdot)$.

When $0<x \leq c_{0}, j(x) \equiv x\left[\left(\ln H^{\prime}(x)\right)^{\prime}-\left(\ln F^{\prime}(x)\right)^{\prime}\right]=a$. Hence

$$
\ln H^{\prime}(x)-\ln F^{\prime}(x)=\bar{k}+a \ln x
$$

for some constant $\bar{k}$, or

$$
H^{\prime}(x)=k F^{\prime}(x) x^{a}=\frac{k}{\sqrt{2 \pi} \sigma} x^{a-1} e^{-(\ln x-\mu)^{2} /\left(2 \sigma^{2}\right)}, \quad 0<x \leq c_{0},
$$

for some constant $k$. Thus,

$$
\begin{aligned}
H(x) & =\frac{k}{\sqrt{2 \pi} \sigma} \int_{0}^{x} t^{a-1} e^{-(\ln t-\mu)^{2} /\left(2 \sigma^{2}\right)} d t \\
& =\frac{k}{\sqrt{2 \pi} \sigma} \int_{-\infty}^{\ln x} e^{a s} e^{-(s-\mu)^{2} /\left(2 \sigma^{2}\right)} d s \\
& =\frac{k}{\sqrt{2 \pi} \sigma} e^{a \mu+a^{2} \sigma^{2} / 2} \int_{-\infty}^{\ln x} e^{-\left(s-\left(\mu+a \sigma^{2}\right)\right)^{2} /\left(2 \sigma^{2}\right)} d s \\
& =k e^{a \mu+a^{2} \sigma^{2} / 2} N\left(\frac{\ln x-\left(\mu+a \sigma^{2}\right)}{\sigma}\right), 0<x \leq c_{0} .
\end{aligned}
$$

Consequently,

$$
T_{+}(z) \equiv H\left(F^{-1}(z)\right)=k e^{a \mu+a^{2} \sigma^{2} / 2} N\left(N^{-1}(z)-a \sigma\right), \quad 0<z \leq F\left(c_{0}\right):=z_{0} .
$$

When $x>c_{0}$, similar to (27) we have

$$
H^{\prime}(x)=\frac{\tilde{k}}{\sqrt{2 \pi} \sigma} x^{b-1} e^{-(\ln x-\mu)^{2} /\left(2 \sigma^{2}\right)}, \quad x>c_{0},
$$

with $\tilde{k}=c_{0}^{a-b} k$ (to render $H^{\prime}(x)$ continuous at $\left.x=c_{0}\right)$. Therefore,

$$
\begin{aligned}
& H(x)=H\left(c_{0}\right)+\frac{\tilde{k}}{\sqrt{2 \pi} \sigma} \int_{c_{0}}^{x} t^{b-1} e^{-(\ln t-\mu)^{2} /\left(2 \sigma^{2}\right)} d t \\
& =H\left(c_{0}\right)+\frac{\tilde{k}}{\sqrt{2 \pi} \sigma} \int_{\ln c_{0}}^{\ln x} e^{b s} e^{-(s-\mu)^{2} /\left(2 \sigma^{2}\right)} d s \\
& =H\left(c_{0}\right)+\frac{\tilde{k}}{\sqrt{2 \pi} \sigma} e^{b \mu+b^{2} \sigma^{2} / 2} \int_{\ln c_{0}}^{\ln x} e^{-\left(s-\left(\mu+b \sigma^{2}\right)\right)^{2} /\left(2 \sigma^{2}\right)} d s \\
& =H\left(c_{0}\right)+\tilde{k} e^{b \mu+b^{2} \sigma^{2} / 2}\left[N\left(\frac{\ln x-\left(\mu+b \sigma^{2}\right)}{\sigma}\right)-N\left(\frac{\ln c_{0}-\left(\mu+b \sigma^{2}\right)}{\sigma}\right)\right], x>c_{0} \text {. }
\end{aligned}
$$


This leads to

$$
\begin{aligned}
& T_{+}(z)=H\left(F^{-1}(z)\right) \\
= & k e^{a \mu+a^{2} \sigma^{2} / 2} N\left(N^{-1}\left(z_{0}\right)-a \sigma\right)+\tilde{k} e^{b \mu+b^{2} \sigma^{2} / 2}\left[N\left(N^{-1}(z)-b \sigma\right)-N\left(N^{-1}\left(z_{0}\right)-a \sigma\right)\right], \\
& z_{0}<z \leq 1 .
\end{aligned}
$$

In particular,

$$
\begin{aligned}
T_{+}(1) & =k e^{a \mu+a^{2} \sigma^{2} / 2} N\left(N^{-1}\left(z_{0}\right)-a \sigma\right)+\tilde{k} e^{b \mu+b^{2} \sigma^{2} / 2}\left[1-N\left(N^{-1}\left(z_{0}\right)-a \sigma\right)\right] \\
& =k e^{a \mu+a^{2} \sigma^{2} / 2} N\left(\frac{\ln c_{0}-\mu-a \sigma^{2}}{\sigma}\right)+k c_{0}^{a-b} e^{b \mu+b^{2} \sigma^{2} / 2}\left[1-N\left(\frac{\ln c_{0}-\mu-a \sigma^{2}}{\sigma}\right)\right] .
\end{aligned}
$$

This, in turn, determines uniquely the value of $k$ since $T_{+}(1)=1$.

So, in this example we have constructed a class of distortions $T_{+}$parameterized by $z_{0}=$ $F^{-1}\left(c_{0}\right) \in(0,1), a<0$ and $b \in(0,1)$. These distortions are reversed S-shaped, and satisfy the monotonicity condition in Assumption 4.1.

The expressions of $H(\cdot)$ given in (28) and (30) show that the distortion $T_{+}(\cdot)$ in effect distorts the distribution of $\rho$, a lognormal random variable, into one having lognormal components, albeit with enlarged means and rescaled values. On the other hand, as stipulated in Tversky and Kahneman (1992), a probability distortion on gain usually satisfies $T_{+}^{\prime}(0)=T_{+}^{\prime}(1)=+\infty$, reflecting the observation that there are most significant distortions on very small and very large probabilities. It turns out that the distortion functions constructed in the preceding example do indeed satisfy $T_{+}^{\prime}(0)=T_{+}^{\prime}(1)=+\infty$. To see this, notice $T_{+}^{\prime}(z)=T_{+}^{\prime}(F(x))=H^{\prime}(x) / F^{\prime}(x)=k x^{j(x)}$ or $\tilde{k} x^{j(x)}$. Hence, when $z \rightarrow 0, x \rightarrow 0$, and $T_{+}^{\prime}(z) \rightarrow+\infty$. On the other hand, when $z \rightarrow 1, x \rightarrow+\infty$, and $T_{+}^{\prime}(z) \rightarrow+\infty$.

\section{Negative Part Problem}

Now we turn to the negative part problem (12), which is a Choquet minimization problem. Such a problem in a more general setting is solved thoroughly in Appendix D; so we need only to apply the results there to (12). Notice, though, (12) has a constraint that a feasible solution must be almost surely bounded from above. The reason we do not include this constraint explicitly into the general problem (51) is that, under a mild condition, any optimal solution to (51) is automatically almost surely bounded from above; see Proposition D.2 and the comments right after it.

Similarly with the positive part problem, for a given $A=\{\omega: \rho \leq c\}$ with $\underline{\rho} \leq c<\bar{\rho}$ (the case when $c=\bar{\rho}$ is trivial), we define $T_{A^{C}}(x):=\frac{T_{-}\left(x P\left(A^{C}\right)\right)}{T_{-}\left(P\left(A^{C}\right)\right)}$. Then $T_{A^{C}}(\cdot)$ is a strictly increasing, differentiable function from $[0,1]$ to $[0,1]$ with $T_{A^{C}}(0)=0, T_{A^{C}}(1)=1$. Moreover, for any feasible solution $X$ of (12) and any $y \geq 0$,

$T_{-}\left(P\left\{u_{-}(X)>y\right\}\right)=T_{-}\left(P\left\{u_{-}(X)>y \mid A^{C}\right\} P\left(A^{C}\right)\right)=T_{-}\left(P\left(A^{C}\right)\right) T_{A^{C}}\left(P\left\{u_{-}(X)>y \mid A^{C}\right\}\right)$. 
Define the probability measure $P_{A^{C}}(\cdot)=P\left(\cdot \mid A^{C}\right)$. Then Problem (12), taken in the probability space $\left(\Omega \cap A^{C}, \mathcal{F} \cap A^{C}, P_{A^{C}}\right)$, is equivalent to

$$
\begin{array}{ll}
\text { Minimize } & V_{-}(Y)=T_{-}\left(P\left(A^{C}\right)\right) \int_{0}^{+\infty} T_{A^{C}}\left(P_{A^{C}}\left\{u_{-}(Y)>y\right\}\right) d y \\
\text { subject to } & E_{A^{C}}[\rho Y]=\left(x_{+}-x_{0}\right) / P\left(A^{C}\right), \quad Y \geq 0, \quad Y \text { is bounded a.s.. }
\end{array}
$$

This is a special case of (51) in Appendix D.

Theorem 7.1 Assume that $u_{-}(\cdot)$ is strictly concave at 0 . Given $A:=\{\omega: \rho \leq c\}$ with $\underline{\rho} \leq c \leq \bar{\rho}$, and $x_{+} \geq x_{0}^{+}$.

(i) If $c=\bar{\rho}$ and $x_{+}=x_{0}$, then the optimal solution of (12) is $X^{*}=0$ and $v_{-}\left(c, x_{+}\right)=0$.

(ii) If $c=\bar{\rho}$ and $x_{+} \neq x_{0}$, then there is no feasible solution to (12) and $v_{-}\left(c, x_{+}\right)=+\infty$.

(iii) If $\underline{\rho} \leq c<\bar{\rho}$, then $v_{-}\left(c, x_{+}\right)=\inf _{\bar{c} \in[c, \bar{\rho})} u_{-}\left(\frac{x_{+}-x_{0}}{E\left[\rho \mathbf{1}_{\rho>\bar{c}}\right]}\right) T_{-}(1-F(\bar{c}))$. Moreover, Problem (12) with parameters $\left(A, x_{+}\right)$admits an optimal solution $X^{*}$ if and only if the following minimization problem

$$
\min _{\bar{c} \in[c, \bar{\rho})} u_{-}\left(\frac{x_{+}-x_{0}}{E\left[\rho \mathbf{1}_{\rho>\bar{c}}\right]}\right) T_{-}(1-F(\bar{c}))
$$

admits an optimal solution $\bar{c}^{*}$, in which case $X^{*}=\frac{x_{+}-x_{0}}{E\left[\rho \mathbf{1}_{\rho>\bar{c}^{*}}\right]} \mathbf{1}_{\rho>\bar{c}^{*}}$, a.s..

Proof: Cases (i) and (ii) are trivial. On the other hand, given Theorem D.1, and noticing that $X^{*}=\frac{x_{+}-x_{0}}{E\left[\rho \mathbf{1}_{\rho>\bar{c}^{*}}\right]} \mathbf{1}_{\bar{c}^{*} \leq \rho<c}$ is automatically bounded, we can prove (iii) similarly to that for Theorem 6.1.

Q.E.D.

\section{Proof of Main Results}

Now that we have solved the problems of the positive and negative parts in Step 1, we are ready to solve our ultimate Problem (7) via the optimization Problem (13) or equivalently, Problem (21), in Step 2, and hence prove the main results contained in Theorem 4.1.

Recall problem (14) formulated earlier. The following lemma is straightforward by Theorem 7.1 and the convention (15).

Lemma 8.1 For any feasible pair $\left(c, x_{+}\right)$for Problem (21), $u_{-}\left(\frac{x_{+}-x_{0}}{E\left[\rho \mathbf{1}_{\rho>c}\right]}\right) T_{-}(1-F(c)) \geq$ $v_{-}\left(c, x_{+}\right)$.

Proposition 8.1 Problems (21) and (14) have the same supremum values.

Proof: Denote by $\alpha$ and $\beta$ the supremum values of (21) and (14) respectively. By Lemma 8.1, $\alpha \geq \beta$. Conversely, we prove $\alpha \leq \beta$. First we assume that $\alpha<+\infty$. For any $\epsilon>0$, 
there exists $\left(c, x_{+}\right)$feasible for $(21)$ such that $v_{+}\left(c, x_{+}\right)-v_{-}\left(c, x_{+}\right) \geq \alpha-\epsilon$, and there exists $\bar{c} \in[c, \bar{\rho}]$ such that $u_{-}\left(\frac{x_{+}-x_{0}}{E\left[\rho \mathbf{1}_{\rho>\bar{c}}\right]}\right) T_{-}(1-F(\bar{c})) \leq v_{-}\left(c, x_{+}\right)+\epsilon$. Therefore

$$
\begin{aligned}
v_{+}\left(\bar{c}, x_{+}\right)-u_{-}\left(\frac{x_{+}-x_{0}}{E\left[\rho \mathbf{1}_{\rho>\bar{c}}\right]}\right) T_{-}(1-F(\bar{c})) & \geq v_{+}\left(\bar{c}, x_{+}\right)-v_{-}\left(c, x_{+}\right)-\epsilon \\
& \geq v_{+}\left(c, x_{+}\right)-v_{-}\left(c, x_{+}\right)-\epsilon \\
& \geq \alpha-2 \epsilon .
\end{aligned}
$$

Letting $\epsilon \rightarrow 0$, we conclude $\alpha \leq \beta$.

Next, if $\alpha=+\infty$, then for any $M \in \mathbb{R}$, there exists a feasible pair $\left(c, x_{+}\right)$such that $v_{+}\left(c, x_{+}\right)-v_{-}\left(c, x_{+}\right) \geq M$, and there is $\bar{c} \geq c$ with $u_{-}\left(\frac{x_{+}-x_{0}}{E\left[\rho \mathbf{1}_{\rho>\bar{c}}\right]}\right) T_{-}(1-F(\bar{c})) \leq v_{-}\left(c, x_{+}\right)+M / 2$. Thus $v_{+}\left(\bar{c}, x_{+}\right)-u_{-}\left(\frac{x_{+}-x_{0}}{E\left[\rho \mathbf{1}_{\rho>\bar{c}}\right.}\right) T_{-}(1-F(\bar{c})) \geq v_{+}\left(c, x_{+}\right)-v_{-}\left(c, x_{+}\right)-M / 2 \geq M / 2$, which implies that $\beta=+\infty$.

Q.E.D.

Proof of Theorem 4.1: (i) If $X^{*}$ is optimal for $(7)$, then by Theorem $5.2\left(c^{*}, x_{+}^{*}\right)$ is optimal for (21) and $\left(X^{*}\right)^{+} \mathbf{1}_{\rho \leq c^{*}}$ and $\left(X^{*}\right)^{-} \mathbf{1}_{\rho>c^{*}}$ are respectively optimal for Problems (11) and (12) with parameters $\left(\left\{\omega: \rho \leq c^{*}\right\}, x_{+}^{*}\right)$. We now show that with $\left(c, x_{+}\right)=\left(c^{*}, x_{+}^{*}\right)$ the minimum in (32) is achieved at $\bar{c}=c^{*}$, namely,

$$
v_{-}\left(c^{*}, x_{+}^{*}\right)=u_{-}\left(\frac{x_{+}^{*}-x_{0}}{E\left[\rho \mathbf{1}_{\rho>c^{*}}\right]}\right) T_{-}\left(1-F\left(c^{*}\right)\right) .
$$

To this end, we first assume that $x_{+}^{*}=0$ (hence $X^{*}=0$ a.s. and $c^{*}=\bar{\rho}$ ). Then $x_{0} \leq x_{+}^{*}=0$. If $x_{0}=0$, then (33) is trivial. If $x_{0}<0$, Theorem 7.1 yields that $\left(X^{*}\right)^{-} \mathbf{1}_{\rho>c^{*}}$ has the following representation

$$
\left(X^{*}\right)^{-} \mathbf{1}_{\rho>c^{*}}=\frac{x_{+}^{*}-x_{0}}{E\left[\rho \mathbf{1}_{\rho>\bar{c}^{*}}\right]} \mathbf{1}_{\rho>\bar{c}^{*}}, \quad \text { a.s. }
$$

Recall $X^{*}<0$ on $\rho>c^{*}$, and $\frac{x_{+}^{*}-x_{0}}{E\left[\rho \mathbf{1}_{\rho>\bar{c}^{*}}\right]}>0$; so (34) implies $c^{*}=\bar{c}^{*}$, and hence (33), in view of Theorem 7.1 .

Next, if $x_{+}^{*}>0$, then by Proposition 6.1 , we have $v_{+}\left(\bar{c}, x_{+}^{*}\right)>v_{+}\left(c^{*}, x_{+}^{*}\right)$ for any $\bar{c}>c^{*}$ with $P\left\{c^{*}<\rho \leq \bar{c}\right\}>0$. If (33) is not true, then it follows from Theorem 7.1 that there exists $\bar{c}>c^{*}$ with $P\left\{c^{*}<\rho \leq \bar{c}\right\}>0$ such that $v_{-}\left(c^{*}, x_{+}^{*}\right)=u_{-}\left(\frac{x_{+}^{*}-x_{0}}{E\left[\rho \mathbf{1}_{\rho>\bar{c}}\right]}\right) T_{-}(1-F(\bar{c}))$. Consequently,

$$
\begin{aligned}
v_{+}\left(\bar{c}, x_{+}^{*}\right)-v_{-}\left(\bar{c}, x_{+}^{*}\right) & \geq v_{+}\left(\bar{c}, x_{+}^{*}\right)-u_{-}\left(\frac{x_{+}^{*}-x_{0}}{E\left[\rho \mathbf{1}_{\rho>\bar{c}}\right]}\right) T_{-}(1-F(\bar{c})) \\
& >v_{+}\left(c^{*}, x_{+}^{*}\right)-v_{-}\left(c^{*}, x_{+}^{*}\right),
\end{aligned}
$$

violating the conclusion that $\left(c^{*}, x_{+}^{*}\right)$ is optimal for $(21)$.

Now, for any $\left(c, x_{+}\right)$feasible for $(14)$,

$$
\begin{aligned}
& v_{+}\left(c, x_{+}\right)-u_{-}\left(\frac{x_{+}-x_{0}}{E\left[\rho \mathbf{1}_{\rho>c}\right]}\right) T_{-}(1-F(c)) \\
\leq & v_{+}\left(c, x_{+}\right)-v_{-}\left(c, x_{+}\right) \\
\leq & v_{+}\left(c^{*}, x_{+}^{*}\right)-v_{-}\left(c^{*}, x_{+}^{*}\right) \\
= & v_{+}\left(c^{*}, x_{+}^{*}\right)-u_{-}\left(\frac{x_{+}^{*}-x_{0}}{E\left[\rho \mathbf{1}_{\rho>c^{*}}\right]}\right) T_{-}\left(1-F\left(c^{*}\right)\right),
\end{aligned}
$$


implying that $\left(c^{*}, x_{+}^{*}\right)$ is optimal for $(14)$. The other conclusions are straightforward.

(ii) Since $\left(c^{*}, x_{+}^{*}\right)$ is optimal for $(14)$, we have

$$
\begin{aligned}
v_{+}\left(c^{*}, x_{+}^{*}\right)-v_{-}\left(c^{*}, x_{+}^{*}\right) & \geq v_{+}\left(c^{*}, x_{+}^{*}\right)-u_{-}\left(\frac{x_{+}^{*}-x_{0}}{E\left[\rho \mathbf{1}_{\left.\rho>c^{*}\right]}\right.}\right) T_{-}\left(1-F\left(c^{*}\right)\right) \\
& =\sup \left[v_{+}\left(c, x_{+}\right)-u_{-}\left(\frac{x_{+}-x_{0}}{E\left[\rho \mathbf{1}_{\rho>c}\right]}\right) T_{-}(1-F(c))\right] \\
& =\sup \left[v_{+}\left(c, x_{+}\right)-v_{-}\left(c, x_{+}\right)\right]
\end{aligned}
$$

where the supremum is over the feasible region of $(14)$. This implies that $\left(c^{*}, x_{+}^{*}\right)$ is optimal for (21) and the inequality above is in fact an equality, resulting in

$$
v_{-}\left(c^{*}, x_{+}^{*}\right)=u_{-}\left(\frac{x_{+}^{*}-x_{0}}{E\left[\rho \mathbf{1}_{\rho>c^{*}}\right]}\right) T_{-}\left(1-F\left(c^{*}\right)\right) \text {. }
$$

The above in turn indicates, thanks to Theorem 7.1 , that $X_{-}^{*}:=\frac{x_{+}^{*}-x_{0}}{E\left[\rho \mathbf{1}_{\rho>c^{*}}\right]} \mathbf{1}_{\rho>c^{*}}$ is optimal for (12) with parameters $\left(\left\{\omega: \rho \leq c^{*}\right\}, x_{+}^{*}\right)$. The desired result then follows from Theorem 5.2. Q.E.D.

Other claims in Section 4 on more explicit conclusions under Assumption 4.1 are straightforward by virtue of Theorem 6.1 .

\section{An Example with Two-Piece CRRA Utility Func- tions}

In this section we solve a concrete (and very involved) example to demonstrate the general results obtained in previous section as well as the algorithm presented. The example showcases all the possibilities associated with our behavioral portfolio selection model (6), namely, a model could be ill-posed, or well-posed yet optimal solution not attainable, or well-posed and optimal solution obtainable. When the optimal solutions do exist, we are able to derive explicit terminal payoffs for most of the cases.

In the example, we let $\rho$ follow the lognormal distribution, i.e., $\ln \rho \sim N(\mu, \sigma)$ with $\sigma>0$, and the utility functions be CRRA (constant relative risk aversion), i.e., $u_{+}(x)=x^{\alpha}, u_{-}(x)=$ $k_{-} x^{\alpha}, x \geq 0$, with $k_{-}>0$ and $0<\alpha<1$. (Recall the overall utility function - or value function in the terminology of Tversky and Kahneman - is an S-shaped function.) These functions, also taken in Tversky and Kahneman (1992) with $\alpha=0.88$ and $k_{-}=2.25$, clearly satisfy Assumption 2.3. We do not spell out (neither do we need) the explicit forms of the distortions $T_{+}(\cdot)$ and $T_{-}(\cdot)$ so long as they satisfy Assumption 2.4. In addition, we assume that Assumption 4.1 holds, which is imposed on $T_{+}(\cdot)$. An example of such $T_{+}(\cdot)$ was presented in Example 6.1.

Clearly, $u_{+}^{\prime}(x)=\alpha x^{\alpha-1},\left(u_{+}^{\prime}\right)^{-1}(y)=(y / \alpha)^{1 /(\alpha-1)}, u_{+}\left(\left(u_{+}^{\prime}\right)^{-1}(y)\right)=(y / \alpha)^{\alpha /(\alpha-1)}, \underline{\rho}=0$, $\bar{\rho}=+\infty$, and $F(x)=N((\ln x-\mu) / \sigma)$.

Under this setting, we first want to solve the positive part problem (11) with given $\left(c, x_{+}\right)$, where $0 \leq c \leq+\infty$ and $x_{+} \geq x_{0}^{+}$. The case that $c=0$ is trivial, where necessarily $x_{+}=0$ in 
order to have a feasible problem, and $v_{+}\left(c, x_{+}\right)=0$. So let $c \in(0,+\infty]$. The optimal solution to (11) in this case is

$$
X_{+}^{*}\left(c, x_{+}\right)=\left(u_{+}^{\prime}\right)^{-1}\left(\frac{\lambda\left(c, x_{+}\right) \rho}{T_{+}^{\prime}(F(\rho))}\right) \mathbf{1}_{\rho \leq c}=\left(\frac{\lambda\left(c, x_{+}\right) \rho}{\alpha T_{+}^{\prime}(F(\rho))}\right)^{1 /(\alpha-1)} \mathbf{1}_{\rho \leq c} .
$$

To determine $\lambda\left(c, x_{+}\right)$, denote

$$
\varphi(c):=E\left[\left(\frac{T_{+}^{\prime}(F(\rho))}{\rho}\right)^{1 /(1-\alpha)} \rho \mathbf{1}_{\rho \leq c}\right]>0, \quad 0<c \leq+\infty .
$$

Then the constraint $x_{+}=E\left[\rho X_{+}^{*}\left(c, x_{+}\right)\right]=\varphi(c)\left(\frac{\lambda\left(c, x_{+}\right)}{\alpha}\right)^{1 /(\alpha-1)}$ gives

$$
\lambda\left(c, x_{+}\right)=\alpha\left(\frac{x_{+}}{\varphi(c)}\right)^{\alpha-1}, 0<c \leq+\infty, x_{+} \geq x_{0}^{+}
$$

This in turn determines

$$
X_{+}^{*}\left(c, x_{+}\right)=\frac{x_{+}}{\varphi(c)}\left(\frac{T_{+}^{\prime}(F(\rho))}{\rho}\right)^{1 /(1-\alpha)} \mathbf{1}_{\rho \leq c}, \quad 0<c \leq+\infty, x_{+} \geq x_{0}^{+},
$$

and

$$
\begin{aligned}
v_{+}\left(c, x_{+}\right) & =\left(\frac{x_{+}}{\varphi(c)}\right)^{\alpha} E\left[\left(\frac{\rho}{T_{+}^{\prime}(F(\rho))}\right)^{\alpha /(\alpha-1)-1} \rho \mathbf{1}_{\rho \leq c}\right] \\
& =\left(\frac{x_{+}}{\varphi(c)}\right)^{\alpha} \varphi(c) \\
& =\varphi(c)^{1-\alpha} x_{+}^{\alpha}, \quad 0<c \leq+\infty, x_{+} \geq x_{0}^{+} .
\end{aligned}
$$

Set $\tilde{\varphi}(c)=\left\{\begin{array}{ll}\varphi(c) & \text { if } 0<c \leq+\infty, \\ 0 & \text { if } c=0,\end{array}\right.$ which is a non-decreasing function right continuous at 0. Then Problem (14) specializes to

$$
\begin{array}{ll}
\text { Maximize } & v\left(c, x_{+}\right)=\tilde{\varphi}(c)^{1-\alpha} x_{+}^{\alpha}-\frac{k_{-} T_{-}(1-F(c))}{\left(E\left[\rho \mathbf{1}_{\rho>c}\right]\right)^{\alpha}}\left(x_{+}-x_{0}\right)^{\alpha}, \\
\text { subject to } & \left\{\begin{array}{l}
0 \leq c \leq+\infty, \quad x_{+} \geq x_{0}^{+}, \\
x_{+}=0 \text { when } c=0, x_{+}=x_{0} \text { when } c=+\infty .
\end{array}\right.
\end{array}
$$

When $c>0$ (excluding $+\infty)$ and $x_{+} \geq x_{0}^{+}$, write

$$
v\left(c, x_{+}\right)=\varphi(c)^{1-\alpha}\left[x_{+}^{\alpha}-k(c)\left(x_{+}-x_{0}\right)^{\alpha}\right],
$$

where $k(c):=\frac{k_{-} T_{-}(1-F(c))}{\varphi(c)^{1-\alpha}\left(E\left[\rho \mathbf{1}_{\rho>c}\right]\right)^{\alpha}}>0, \quad c>0$.

We study the underlying portfolio selection problem in two cases, depending on whether the initial wealth represents a gain or a loss.

TheOREM 9.1 Assume that $x_{0} \geq 0$ and Assumption 4.1 holds. 
(i) If $\inf _{c>0} k(c) \geq 1$, then the optimal portfolio for Problem (6) is the replicating portfolio for the contingent claim

$$
X^{*}=\frac{x_{0}}{\varphi(+\infty)}\left(\frac{T_{+}^{\prime}(F(\rho))}{\rho}\right)^{1 /(1-\alpha)} .
$$

(ii) If $\inf _{c>0} k(c)<1$, then Problem (6) is ill-posed.

Proof: Consider the problem $\max _{x \geq x_{0}} f(x)$ where $f(x)=x^{\alpha}-k\left(x-x_{0}\right)^{\alpha}$ and $k \geq 0$ fixed. Since $f^{\prime}(x)=\alpha\left[x^{\alpha-1}-k\left(x-x_{0}\right)^{\alpha-1}\right]$, we conclude that 1) if $k \geq 1$, then $f^{\prime}(x) \leq$ $0 \forall x \geq x_{0}$; therefore $x^{*}=x_{0}$ is optimal with the optimal value $x_{0}^{\alpha}$; and 2) if $k<1$, then $f(x)=x^{\alpha}\left[1-k\left(1-x_{0} / x\right)^{\alpha}\right] \rightarrow+\infty$ as $x \rightarrow+\infty$, implying that $\sup _{x \geq x_{0}} f(x)=+\infty$.

(i) If $\inf _{c>0} k(c) \geq 1$, then

$$
\begin{aligned}
\sup _{c>0, x_{+} \geq x_{0}^{+}} v\left(c, x_{+}\right) & \equiv \sup _{c>0}\left[\varphi(c)^{1-\alpha} \sup _{x_{+} \geq x_{0}}\left(x_{+}^{\alpha}-k(c)\left(x_{+}-x_{0}\right)^{\alpha}\right)\right] \\
& =\sup _{c>0}\left[\varphi(c)^{1-\alpha} x_{0}^{\alpha}\right]=\varphi(+\infty)^{1-\alpha} x_{0}^{\alpha} \equiv v_{+}\left(+\infty, x_{0}\right) \geq 0 .
\end{aligned}
$$

However, when $c=0$ (and hence $x_{+}=0$ ) we have $v\left(c, x_{+}\right)=0$. As a result $\left(c^{*}, x_{+}^{*}\right)=$ $\left(+\infty, x_{0}\right)$ is optimal to $(37)$. Theorem 4.1 then applies to conclude that $X^{*} \equiv X_{+}^{*}\left(+\infty, x_{0}\right)=$ $\frac{x_{0}}{\varphi(+\infty)}\left(\frac{T_{+}^{\prime}(F(\rho))}{\rho}\right)^{1 /(1-\alpha)}$ solves $(7)$. Hence the optimal portfolio for (6) is the one that replicates $X^{*}$.

(ii) If $\inf _{c>0} k(c)<1$, then there is $c_{0}>0$ such that $k\left(c_{0}\right)<1$. In this case,

$$
\sup _{c>0, x_{+} \geq x_{0}^{+}} v\left(c, x_{+}\right) \geq j\left(c_{0}\right)^{\alpha} \sup _{x_{+} \geq x_{0}}\left[x_{+}^{\alpha}-k\left(c_{0}\right)\left(x_{+}-x_{0}\right)^{\alpha}\right]=+\infty .
$$

The conclusion thus follows from Propositions 8.1 and 5.1. .

Q.E.D.

TheOREM 9.2 Assume that $x_{0}<0$ and Assumption 4.1 holds.

(i) If $\inf _{c>0} k(c)>1$, then Problem (6) is well-posed. Moreover, (6) admits an optimal portfolio if and only if

$$
\operatorname{argmin}_{c \geq 0}\left[\left(\frac{k_{-} T_{-}(1-F(c))}{\left(E\left[\rho \mathbf{1}_{\rho>c}\right]\right)^{\alpha}}\right)^{1 /(1-\alpha)}-\tilde{\varphi}(c)\right] \neq \varnothing .
$$

Furthermore, if $c^{*}>0$ is one of the minimizers in (38), then the optimal portfolio is the one to replicate

$$
X^{*}=\frac{x_{+}^{*}}{\varphi\left(c^{*}\right)}\left(\frac{T_{+}^{\prime}(F(\rho))}{\rho}\right)^{1 /(1-\alpha)} \mathbf{1}_{\rho \leq c^{*}}-\frac{x_{+}^{*}-x_{0}}{E\left[\rho \mathbf{1}_{\rho>c^{*}}\right]} \mathbf{1}_{\rho>c^{*}},
$$

where $x_{+}^{*}:=\frac{-x_{0}}{k\left(c^{*}\right)^{1 /(1-\alpha)}-1}$; and if $c^{*}=0$ is the unique minimizer in (38), then the unique optimal portfolio is the one to replicate $X^{*}=\frac{x_{0}}{E \rho}$.

(ii) If $\inf _{c>0} k(c)=1$, then the supremum value of Problem (6) is 0, which is however not achieved by any admissible portfolio. 
(iii) If $\inf _{c>0} k(c)<1$, then Problem (6) is ill-posed.

Proof: We first consider a general optimization problem $\max _{x \geq 0} f(x)$ where $f(x):=$ $x^{\alpha}-k\left(x-x_{0}\right)^{\alpha}$ and $k \geq 0$ fixed. We solve it in the following three cases.

1) If $k>1$, then $f^{\prime}(x)=0$ has the only solution $x^{*}=\frac{-x_{0}}{k^{1 /(1-\alpha)}-1}>0$. Since $f^{\prime \prime}(x)=$ $\alpha(\alpha-1)\left[x^{\alpha-2}-k\left(x-x_{0}\right)^{\alpha-2}\right]<0 \forall x>0, x^{*}$ is the (only) maximum point with the maximum value

$$
f\left(x^{*}\right)=\left(x^{*}\right)^{\alpha}\left[1-k\left(1-x_{0} / x\right)^{\alpha}\right]=-\left(-x_{0}\right)^{\alpha}\left[k^{1 /(1-\alpha)}-1\right]^{1-\alpha} .
$$

2) If $k=1$, then $f^{\prime}(x)>0 \forall x>0$. This means that the supremum of $f(x)$ on $x \geq 0$ is $\lim _{x \rightarrow+\infty} f(x)=0$; yet this value is not achieved by any $x \geq 0$.

3) If $k<1$, then $f(x)=x^{\alpha}\left[1-k\left(1-x_{0} / x\right)^{\alpha}\right] \rightarrow+\infty$ as $x \rightarrow+\infty$, implying that $\sup _{x \geq 0} f(x)=+\infty$.

We need to solve (37) to obtain $\left(c^{*}, x_{+}^{*}\right)$. Since $x_{0}<0, c=+\infty$ is infeasible; so we restrict $c \in[0,+\infty)$. Care must be taken to deal with the special solution $\left(c, x_{+}\right)=(0,0)$ with $v(0,0)=-\frac{k_{-}}{(E[\rho]]^{\alpha}}\left(-x_{0}\right)^{\alpha}$.

(i) If $\inf _{c>0} k(c)>1$, then

$$
\begin{aligned}
\sup _{c>0, x_{+} \geq x_{0}^{+}} v\left(c, x_{+}\right) & \equiv \sup _{c>0}\left[\varphi(c)^{1-\alpha} \sup _{x_{+} \geq 0}\left(x_{+}^{\alpha}-k(c)\left(x_{+}-x_{0}\right)^{\alpha}\right)\right] \\
& =\sup _{c>0}\left[-\left(-x_{0}\right)^{\alpha} \varphi(c)^{1-\alpha}\left(k(c)^{1 /(1-\alpha)}-1\right)^{1-\alpha}\right] \\
& =-\left(-x_{0}\right)^{\alpha}\left\{\inf _{c>0}\left[\left(\frac{k_{-} T_{-}(1-F(c))}{\left(E\left[\rho 1_{\rho>c}\right)^{\alpha}\right.}\right)^{1 /(1-\alpha)}-\varphi(c)\right]\right\}^{1-\alpha}<+\infty .
\end{aligned}
$$

This yields that (6) is well-posed. Now, if $c^{*}>0$ achieve the infimum of $\left[\left(\frac{k_{-} T_{-}(1-F(c))}{\left(E\left[\rho 1_{\rho>c}\right)^{*}\right.}\right)^{1 /(1-\alpha)}-\tilde{\varphi}(c)\right]$ over $c \geq 0$, then we have $\sup _{c>0, x_{+} \geq x_{0}^{+}} v\left(c, x_{+}\right) \geq-\left(-x_{0}\right)^{\alpha} \frac{k_{-}}{(E \rho)^{\alpha}}=v(0,0)$, which means $c^{*}>0$ and $x_{+}^{*}=\frac{-x_{0}}{k\left(c^{*}\right)^{1 /(1-\alpha)}-1}$ are optimal for (37). Theorem 4.1 then yields that the optimal portfolio is the one that replicates $X^{*}$ given by (39).

If $c^{*}=0$ is the unique infimum of $\left[\left(\frac{k_{-} T_{-}(1-F(c))}{\left(E\left[\rho 1_{\rho>c}\right)^{\alpha}\right.}\right)^{1 /(1-\alpha)}-\tilde{\varphi}(c)\right]$ over $c \geq 0$, then

$$
\begin{aligned}
\sup _{c>0, x_{+} \geq x_{0}^{+}} v\left(c, x_{+}\right) & =-\left(-x_{0}\right)^{\alpha}\left\{\inf _{c>0}\left[\left(\frac{k_{-} T_{-}(1-F(c))}{\left(E\left[\rho \mathbf{1}_{\rho>c}\right]\right)^{\alpha}}\right)^{1 /(1-\alpha)}-\varphi(c)\right]\right\}^{1-\alpha} \\
& <-\left(-x_{0}\right)^{\alpha} \frac{k_{-}}{(E \rho)^{\alpha}} \equiv v(0,0) .
\end{aligned}
$$

This implies that $\left(c^{*}, x_{+}^{*}\right)=(0,0)$ is uniquely optimal for $(37)$, and the unique optimal solution for $(7)$ is $X_{+}^{*}\left(c^{*}, x_{+}^{*}\right) \mathbf{1}_{\rho \leq c^{*}}-\frac{x_{+}^{*}-x_{0}}{E\left[\rho 1_{\rho>c^{*}}\right]} \mathbf{1}_{\rho>c^{*}} \equiv \frac{x_{0}}{E \rho}$, for which the corresponding replicating portfolio is the risk-free one.

If the infimum $\inf _{c \geq 0}\left[\left(\frac{k_{-} T_{-}(1-F(c))}{\left(E\left[\rho 1_{\rho>c}\right)^{\alpha}\right.}\right)^{1 /(1-\alpha)}-\tilde{\varphi}(c)\right]$ is not attainable, then $\sup _{c>0, x_{+} \geq x_{0}^{+}} v\left(c, x_{+}\right)>-\frac{k_{-}}{(E[\rho])^{\alpha}}\left(-x_{0}\right)^{\alpha}=v(0,0)$. This means that $(0,0)$ is not optimal for 
(37). On the other hand, the optimality of (37) is not achieved at any $c>0$ and $x_{+} \geq 0$ in view of (40). It then follows from Theorem 4.1 that (6) admits no optimal solution.

(ii) Next consider the case when $\inf _{c>0} k(c)=1$. If $k(c)>1$ for any $c>0$, then

$$
\sup _{c>0, x_{+} \geq x_{0}^{+}} v\left(c, x_{+}\right)=-\left(-x_{0}\right)^{\alpha}\left\{\varphi(c)^{1-\alpha}\left[\left(\inf _{c>0} k(c)\right)^{1 /(1-\alpha)}-1\right]^{1-\alpha}\right\}=0 .
$$

Yet, for any $c>0, x_{+} \geq 0, v\left(c, x_{+}\right) \leq \max _{x_{+} \geq 0} v\left(c, x_{+}\right)=-\left(-x_{0}\right)^{\alpha}\left[\varphi(c)^{1-\alpha}\left(k(c)^{1 /(1-\alpha)}-\right.\right.$ $\left.1)^{1-\alpha}\right]<0$. Also, $v(0,0)<0$. Therefore the optimal value is not attainable.

On the other hand, if there exists $c^{*}>0$ such that $k\left(c^{*}\right)=1$, then $\sup _{c \geq 0, x_{+} \geq x_{0}^{+}} v\left(c, x_{+}\right) \geq$ $\sup _{x_{+} \geq 0} v\left(c^{*}, x_{+}\right)=0$. However, $v\left(c, x_{+}\right)=\varphi(c)^{1-\alpha}\left[x_{+}^{\alpha}-k(c)\left(x_{+}-x_{0}\right)^{\alpha}\right] \leq \varphi(c)^{1-\alpha}\left[x_{+}^{\alpha}-\right.$ $\left.\left(x_{+}-x_{0}\right)^{\alpha}\right]<0 \forall c>0, x_{+} \geq 0$. Together with the fact that $v(0,0)<0$ we conclude that $\sup _{c \geq 0, x_{+} \geq x_{0}^{+}} v\left(c, x_{+}\right)=0$, which is however not achieved.

(iii) If $\inf _{c \geq 0} k(c)<1$, then there exists $c_{0}$ such that $k\left(c_{0}\right)<1$. As a result, $v\left(c_{0}, x_{+}\right)=$ $\varphi\left(c_{0}\right)^{1-\alpha}\left[x_{+}^{\alpha}-k\left(c_{0}\right)\left(x_{+}-x_{0}\right)^{\alpha}\right] \rightarrow+\infty$ as $x_{+} \rightarrow+\infty$.

Q.E.D.

We see that the key features of the underlying behavioral portfolio selection problem critically depend on the value $\inf _{c>0} k(c)$. Recall that $k(c)$, by its definition, reflects in a precise way the coordination among the utility functions, the probability distortions, and the market (represented by $\rho$ ). Let us elaborate on one particular point. In Tversky and Kahneman (1992), the parameters are taken, based on extensive experiments, to be $\alpha=0.88$, and $k_{-}=2.25>1$, the latter reflecting the fact that losses loom larger than gains: the pain associated with a loss is typically larger than the pleasure associated with an equivalent gain $^{10}$. Now, by the definition of $k(c)$ we see the larger the loss aversion the more likely the underlying model is well-posed and solvable. The economic intuition behind this is that with a larger loss aversion coefficient it is not optimal to allocate all the fund to stocks (because stocks are risky and prone to losses), and hence one needs to carefully balance the investment between risky and risk-free assets, leading to a meaningful model.

Another interesting observation is that the optimal portfolios behave fundamentally different depending on whether $x_{0}>0$ (Theorem 9.1) or $x_{0}<0$ (Theorem 9.2). Recall that the state 0 here really means the reference point (e.g., the present value of a future liability that must be fulfilled); therefore the two situations correspond to whether the investor starts with a gain or loss situation. If $x_{0}>0$, then the optimal strategy is simply to spend $x_{0}$ buying a contingent claim that delivers a payoff in excess of the reference point, reminiscent of a classical utility maximizing agent (although the allocation to stocks is "distorted" due to the probability distortion). If $x_{0}<0$, then the investor starts off a loss situation and needs to get "out of the hole" soonest possible. As a result, the optimal strategy is a gambling policy which involves raising additional capital to purchase a claim that delivers a higher payoff in the case of a good state of the market and incurs a fixed loss in the case of a bad one. Finally, if $x_{0}=0$, then the optimal portfolio is not to invest in risky asset at all. Notice that $x_{0}=0$ corresponds to a natural psychological reference point - the risk-free return - for many people. This, nonetheless, does explain why most households do not invest in equities at all ${ }^{11}$.

\footnotetext{
${ }^{10} k_{-}$is the so-called loss aversion coefficient.

${ }^{11}$ A similar result is derived in Gomes (2005) for his portfolio selection model with loss averse investors, albeit in the single-period setting without probability distortions.
} 


\section{How Behavioral Criterion Affects Risky Allocation}

Along the line of the discussions at the end of the last section we would like to investigate more on how exactly the behavioral criterion would affect the wealth allocation to risky assets. This is best explained through a very concrete example, where an optimal portfolio (not just optimal terminal payoff) is explicitly available. We consider a model with the power utility $u_{+}(x)=x^{\alpha}, u_{-}(x)=k_{-} x^{\alpha}$, and all the market parameters (investment opportunity set) are time-invariant: $r(\cdot) \equiv r, B(\cdot)=B, \sigma(t)=\sigma, \theta(\cdot)=\theta$. In this case $\rho(t, T):=\rho(T) / \rho(t)$, given $\mathcal{F}_{t}$, follows a lognormal distribution with parameter $\left(\mu_{t}, \sigma_{t}^{2}\right)$, where

$$
\mu_{t}:=-\left(r+\theta^{2} / 2\right)(T-t), \quad \sigma_{t}^{2}:=\theta^{2}(T-t) .
$$

Furthermore, we set the distortion $T_{+}$to be the one in Example 6.1 with $j(x)=: a \mathbf{1}_{0<x \leq c_{0}}+$ $b \mathbf{1}_{x>c_{0}}$, where $c_{0}>0, a<0$ and $0<b<1$.

We now derive in closed-form the optimal portfolio under the setting of Theorem 9.1-(i), i.e., $x_{0} \geq 0$ and $\inf _{c>0} k(c) \geq 1$. (Other cases can also be done, which are left to interested readers.)

THEOREM 10.1 Under the assumption of Theorem 9.1-(i), the optimal wealth-portfolio pair $\left(x^{*}(\cdot), \pi^{*}(\cdot)\right)$ for Problem (6) is

$$
\begin{aligned}
x^{*}(t) & =\frac{x_{0}}{\gamma}\left[x^{1}(t)+c_{0}^{(a-b) /(1-\alpha)} x^{2}(t)\right], \\
\pi^{*}(t) & =\frac{x_{0}}{\gamma}\left[\frac{(1-a) x^{1}(t)+c_{0}^{(a-b) /(1-\alpha)}(1-b) x^{2}(t)}{1-\alpha}\right]\left(\sigma \sigma^{\prime}\right)^{-1} B,
\end{aligned}
$$

where $\psi(y):=(2 \pi)^{-1 / 2} e^{-y^{2} / 2}$ is the density function of a standard normal distribution, and

$$
\begin{aligned}
& x^{1}(t):=\frac{\rho(t)^{(a-1) /(1-\alpha)}}{\sigma_{t}} \int_{0}^{c_{0} / \rho(t)} y^{(a-1) /(1-\alpha)} \psi\left(\frac{\ln y-\mu_{t}}{\sigma_{t}}\right) d y \equiv \frac{1}{\sigma_{t} \rho(t)} \int_{0}^{c_{0}} y^{(a-1) /(1-\alpha)} \psi\left(\frac{\ln y-\mu_{t}-\ln \rho(t)}{\sigma_{t}}\right) d y, \\
& x^{2}(t):=\frac{\rho(t)^{(b-1) /(1-\alpha)}}{\sigma_{t}} \int_{c_{0} / \rho(t)}^{+\infty} y^{(b-1) /(1-\alpha)} \psi\left(\frac{\ln y-\mu_{t}}{\sigma_{t}}\right) d y \equiv \frac{1}{\sigma_{t} \rho(t)} \int_{c_{0}}^{+\infty} y^{(b-1) /(1-\alpha)} \psi\left(\frac{\ln y-\mu_{t}-\ln \rho(t)}{\sigma_{t}}\right) d y, \\
& \gamma:=E\left[\rho^{(a-\alpha) /(1-\alpha)} \mathbf{1}_{\rho \leq c_{0}}+c_{0}^{(a-b) /(1-\alpha)} \rho^{(b-\alpha) /(1-\alpha)} \mathbf{1}_{\rho>c_{0}}\right] .
\end{aligned}
$$

Proof: It follows from (27) and (29) that

$$
\frac{T_{+}^{\prime}(F(\rho))}{\rho} \equiv \frac{H^{\prime}(\rho)}{\rho F^{\prime}(\rho)}=k \rho^{a-1} \mathbf{1}_{\rho \leq c_{0}}+k c_{0}^{a-b} \rho^{b-1} \mathbf{1}_{\rho>c_{0}},
$$

where $k^{-1}=e^{a \mu_{0}+a^{2} \sigma_{0}^{2} / 2} N\left(\frac{\ln c_{0}-\mu_{0}-a \sigma_{0}^{2}}{\sigma_{0}}\right)+c_{0}^{a-b} e^{b \mu_{0}+b^{2} \sigma_{0}^{2} / 2}\left[1-N\left(\frac{\ln c_{0}-\mu_{0}-a \sigma_{0}^{2}}{\sigma_{0}}\right)\right]$. Hence

$$
\varphi(+\infty):=E\left[\left(\frac{T_{+}^{\prime}(F(\rho))}{\rho}\right)^{\frac{1}{1-\alpha}} \rho\right]=k^{\frac{1}{1-\alpha}} E\left[\rho^{\frac{a-\alpha}{1-\alpha}} \mathbf{1}_{\rho \leq c_{0}}+c_{0}^{\frac{a-b}{1-\alpha}} \rho^{\frac{b-\alpha}{1-\alpha}} \mathbf{1}_{\rho>c_{0}}\right]=k^{\frac{1}{1-\alpha}} \gamma .
$$

Appealing to Theorem 9.1-(i) the optimal portfolio is the replicating portfolio for the claim

$$
X^{*}=\frac{x_{0}}{\gamma}\left[\rho^{(a-1) /(1-\alpha)} \mathbf{1}_{\rho \leq c_{0}}+c_{0}^{(a-b) /(1-\alpha)} \rho^{(b-1) /(1-\alpha)} \mathbf{1}_{\rho>c_{0}}\right] .
$$


Let $\left(x^{1}(\cdot), \pi^{1}(\cdot)\right)$ replicate $\rho^{(a-1) /(1-\alpha)} \mathbf{1}_{\rho \leq c_{0}}$ and $\left(x^{2}(\cdot), \pi^{2}(\cdot)\right)$ replicate $\rho^{(b-1) /(1-\alpha)} \mathbf{1}_{\rho>c_{0}}$. Then the results in Appendix B yield

$$
\begin{aligned}
x^{1}(t) & =\frac{\rho(t)^{(a-1) /(1-\alpha)}}{\sigma_{t}} \int_{0}^{c_{0} / \rho(t)} y^{(a-1) /(1-\alpha)} \psi\left(\frac{\ln y-\mu_{t}}{\sigma_{t}}\right) d y, \\
\pi^{1}(t) & =-\left[\frac{a-1}{1-\alpha} x^{1}(t)-\frac{1}{\sigma_{t} \rho(t)} c_{0}^{(a-\alpha) /(1-\alpha)} \psi\left(\frac{\ln c_{0}-\mu_{t}-\ln \rho(t)}{\sigma_{t}}\right)\right]\left(\sigma \sigma^{\prime}\right)^{-1} B, \\
x^{2}(t) & =\frac{\rho(t)^{(b-1) /(1-\alpha)}}{\sigma_{t}} \int_{c_{0} / \rho(t)}^{+\infty} y^{(b-1) /(1-\alpha)} \psi\left(\frac{\ln y-\mu_{t}}{\sigma_{t}}\right) d y, \\
\pi^{2}(t) & =-\left[\frac{b-1}{1-\alpha} x^{2}(t)+\frac{1}{\sigma_{t} \rho(t)} c_{0}^{(b-\alpha) /(1-\alpha)} \psi\left(\frac{\ln c_{0}-\mu_{t}-\ln \rho(t)}{\sigma_{t}}\right)\right]\left(\sigma \sigma^{\prime}\right)^{-1} B .
\end{aligned}
$$

Combining these two portfolios linearly we obtain the desired result.

Q.E.D.

Now consider the case when $c_{0}=1$ for ease of exposition. In this case the optimal portfolio can be simplified to be

$$
\begin{aligned}
\pi^{*}(t) & =\frac{x_{0}}{\gamma}\left[\frac{(1-a) x^{1}(t)+(1-b) x^{2}(t)}{1-\alpha}\right]\left(\sigma \sigma^{\prime}\right)^{-1} B \\
& =\frac{(1-a) x^{1}(t)+(1-b) x^{2}(t)}{x^{1}(t)+x^{2}(t)} \frac{x^{*}(t)}{1-\alpha}\left(\sigma \sigma^{\prime}\right)^{-1} B \\
& =\left(1-\frac{a x^{1}(t)+b x^{2}(t)}{x^{1}(t)+x^{2}(t)}\right) \frac{x^{*}(t)}{1-\alpha}\left(\sigma \sigma^{\prime}\right)^{-1} B,
\end{aligned}
$$

or the optimal ratio in risky assets is

$$
\frac{\pi^{*}(t)}{x^{*}(t)}=(1-\alpha)^{-1}\left(1-\frac{a x^{1}(t)+b x^{2}(t)}{x^{1}(t)+x^{2}(t)}\right)\left(\sigma \sigma^{\prime}\right)^{-1} B .
$$

Recall that in the conventional expected utility model with the utility function $u(x)=x^{\alpha}$ and without distortion, the optimal ratio in risky assets is

$$
\frac{\hat{\pi}(t)}{\hat{x}(t)}=(1-\alpha)^{-1}\left(\sigma \sigma^{\prime}\right)^{-1} B
$$

So when

$$
\frac{b}{-a}>\frac{x^{1}(t)}{x^{2}(t)}=\frac{\int_{0}^{1} y^{(a-1) /(1-\alpha)} \psi\left(\frac{\ln y-\mu_{t}-\ln \rho(t)}{\sigma_{t}}\right) d y}{\int_{1}^{+\infty} y^{(b-1) /(1-\alpha)} \psi\left(\frac{\ln y-\mu_{t}-\ln \rho(t)}{\sigma_{t}}\right) d y}
$$

the investor underweights the risky assets in her portfolio compared with the one dictated by the conventional utility model, and vice versa.

\section{Concluding Remarks}

In this paper, we introduce, for the first time in literature to our best knowledge, a general continuous-time portfolio selection model within the framework of the cumulative prospect 
theory, so as to account for human psychology and emotions in investment activities. The model features inherent difficulties, including non-convex/concave and non-smooth (overall) utility functions and probability distortions. Even the well-posedness of such a model becomes more an exception than a rule: we demonstrate that a well-posed model calls for a careful coordination among the underlying market, the utility function, and the probability distortions. We then develop an approach to solving the model thoroughly. The approach is largely different from the existing ones employed in the conventional dynamic asset allocation models. Notwithstanding the complexity of the approach, the final solution turns out to be simply structured: the optimal terminal payoff is related to certain binary options characterized by a single number, and the optimal strategy is an aggressive gambling policy betting on good states of the market. Finally, we apply the general results to a specific case with a two-piece CRRA utility function, and show how the behavioral criterion will change the risky allocation.

The equity premium puzzle [Mehra and Prescott (1985)] refers to the phenomenon that observed average annual returns on stocks over the past century are higher by large margin (approximately 6 percentage points) than returns on government bonds, whereas standard asset allocation theories (such as that based on the utility model) predict that the difference in returns between these two investments should be much smaller. Benartzi and Thaler (1995) proposed an explanation for the puzzle using prospect theory (in single period and without probability distortion). In Section 10 we demonstrate that the investor would indeed underweight stocks in her portfolio under certain conditions. We are not claiming that we have provided a satisfactory explanation to the equity premium puzzle in the continuous time setting; but we do hope that the research along the line will shed lights on eventually solving the puzzle.

It should be emphasized again that the agent under study in this paper is a "small investor" in that his behavior will not affect the market. Hence we can still comfortably assume some market properties, such as the absence of arbitrage and the market completeness, as usually imposed for the conventional utility model. (It remains an interesting problem to study a behavioral model in an incomplete market.) It is certainly a fascinating and challenging problem to study how the overall market might be changed by the joint behaviors of investors; e.g., a "behavioral" capital asset pricing model.

Let us also mention about an on-going work [He and Zhou (2007)] on behavioral portfolio choice in single period, featuring both S-shaped utilities and probability distortions. Perversely, the single-period model is equally difficult, and calls for a technique quite different from its continuous-time counterpart to tackle. Only some special cases have been solved, which are used to study the equity premium puzzle more closely.

To conclude, this work is meant to be initiating and inspiring, rather than exhaustive and conclusive, for the research on intertemporal behavioral portfolio allocation. 


\section{Appendix}

\section{A An Inequality}

Lemma A.1 Let $f: \mathbb{R}^{+} \mapsto \mathbb{R}^{+}$be a non-decreasing function with $f(0)=0$. Then

$$
x y \leq \int_{0}^{x} f^{-1}(t) d t+\int_{0}^{y} f(t) d t \quad \forall x \geq 0, y \geq 0,
$$

and the equality holds if and only if $f(y-) \leq x \leq f(y+)$.

Proof: By interpreting the integrations involved as the appropriate areas, we have

$$
\int_{0}^{y} f(t) d t=y f(y)-\int_{0}^{f(y)} f^{-1}(t) d t .
$$

Define $g(x, y):=\int_{0}^{x} f^{-1}(t) d t+\int_{0}^{y} f(t) d t-x y$. Then

$$
g(x, y)=y(f(y)-x)+\int_{f(y)}^{x} f^{-1}(t) d t=\int_{f(y)}^{x}\left(f^{-1}(t)-y\right) d t .
$$

We now consider all the possible cases. First, if $x<f(y-)$, then $f^{-1}(t) \leq y \forall t<f(y)$. Therefore $g(x, y)=\int_{x}^{f(y)}\left(y-f^{-1}(t)\right) d t \geq 0$. Moreover, in this case there exists $z>x$ such that $z<f(y-)$, which implies $y>f^{-1}(z)$ (otherwise $f(y-\epsilon)<z \forall \epsilon>0$, leading to $z \geq f(y-)$ ). The monotonicity of $f^{-1}$ yields $y>f^{-1}(t)$ for any $t \leq z$. Hence $g(x, y)=\int_{x}^{f(y)}\left(y-f^{-1}(t)\right) d t \geq \int_{x}^{z}\left(y-f^{-1}(t)\right) d t>0$.

Next consider the case when $x \in[f(y-), f(y)]$. Since $f^{-1}(t) \leq y \forall t<f(y)$, and $f^{-1}(t) \geq y$ $\forall t>x \geq f(y-)$, we have $g(x, y)=\int_{x}^{f(y)}\left(y-f^{-1}(t)\right) d t=0$.

Symmetrically, we can prove that $g(x, y)=0$ when $x \in[f(y), f(y+)]$, and $g(x, y)>0$ when $x>f(y+)$. The proof is complete.

Q.E.D.

\section{B Two Auxiliary Optimization Problems}

In this subsection we solve two auxiliary optimization problems, which play a key role in simplifying the behavioral portfolio selection model.

Let $Y$ be a given strictly positive random variable on $(\Omega, \mathcal{F}, P)$ with the probability distribution function $F(\cdot)$. Let $G(\cdot)$ be another given distribution function with $G(0)=0$. Consider the following two optimization problems:

$$
\begin{array}{ll}
\text { Maximize } & E[X Y] \\
\text { subject to } & P(X \leq x)=G(x) \quad \forall x \in \mathbb{R},
\end{array}
$$

and

$$
\begin{array}{ll}
\text { Minimize } & E[X Y] \\
\text { subject to } & P(X \leq x)=G(x) \forall x \in \mathbb{R} .
\end{array}
$$

These are two highly non-convex optimization problems.

Lemma B.1 (i) Let $h(\cdot)$ be a non-decreasing function. If $X$ and $h(Y)$ share the same distribution, then $E[X Y] \leq E[h(Y) Y]$ while the equality holds if and only if $X \in[h(Y-), h(Y+)]$ a.s.. 
(ii) Let $h(\cdot)$ be a non-increasing function. If $X$ and $h(Y)$ share the same distribution, then $E[X Y] \geq E[h(Y) Y]$ while the equality holds if and only if $X \in[h(Y-), h(Y+)]$ a.s.

Proof: (i) First assume $h(0)=0$. Employing Lemma A.1, together with the assumption that $X$ and $h(Y)$ have the same distribution, we have

$$
\begin{aligned}
E[X Y] & \leq E\left[\int_{0}^{X} h^{-1}(u) d u\right]+E\left[\int_{0}^{Y} h(u) d u\right] \\
& =E\left[\int_{0}^{h(Y)} h^{-1}(u) d u\right]+E\left[\int_{0}^{Y} h(u) d u\right]=E[h(Y) Y],
\end{aligned}
$$

and the equality holds if and only if $X \in[h(Y-), h(Y+)]$ a.s..

For the general case when $h(0) \neq 0$, define $\bar{h}(x):=h(x)-h(0)$. Then

$$
E[X Y]=E[(X-h(0)) Y]+h(0) E Y \leq E[\bar{h}(Y) Y]+h(0) E Y=E[h(Y) Y] .
$$

(ii) It is straightforward by applying the result in (i) to $-X$ and $-h(Y)$ Q.E.D.

Theorem B.1 Assume that $Y$ admits no atom.

(i) Define $X_{1}^{*}:=G^{-1}(F(Y))$. Then $E\left[X_{1}^{*} Y\right] \geq E[X Y]$ for any feasible solution $X$ of Problem (44). If in addition $E\left[X_{1}^{*} Y\right]<+\infty$, then $X_{1}^{*}$ is the unique (in the sense of almost surely) optimal solution for (44).

(ii) Define $X_{2}^{*}:=G^{-1}(1-F(Y))$. Then $E\left[X_{2}^{*} Y\right] \leq E[X Y]$ for any feasible solution $X$ of Problem (45). If in addition $E\left[X_{2}^{*} Y\right]<+\infty$, then $X_{2}^{*}$ is the unique optimal solution for (45).

Proof: First of all note that $Z:=F(Y)$ follows uniform distribution on the (open or closed) unit interval.

(i) Define $h_{1}(x):=G^{-1}(F(x))$. Then $P\left\{h_{1}(Y) \leq x\right\}=P\{Z \leq G(x)\}=G(x)$, and $h_{1}(\cdot)$ is non-decreasing. By Lemma B.1, $E\left[X_{1}^{*} Y\right] \geq E[X Y]$ for any feasible solution $X$ of Problem (44), where $X_{1}^{*}:=h_{1}(Y)$. Furthermore, if $E\left[X_{1}^{*} Y\right]<+\infty$, and there is $X$ which is optimal for (44), then $E[X Y]=E\left[X_{1}^{*} Y\right]$. By Lemma B.1, $X \in\left[h_{1}(Y-), h_{1}(Y+)\right]$ a.s.. Since $h_{1}(\cdot)$ is non-decreasing, its set of discontinuous points is at most countable. However, $Y$ admits no atom; hence $h_{1}(Y-)=$ $h_{1}(Y+)=h_{1}(Y)$, a.s., which implies that $X=h_{1}(Y)=X_{1}^{*}$, a.s.. Therefore we have proved that $X_{1}^{*}$ is the unique optimal solution for (44).

(ii) Define $h_{2}(x):=G^{-1}(1-F(x))$. It is immediate that $P\left\{h_{2}(Y) \leq x\right\}=G(x)$, and $h_{2}(\cdot)$ is non-increasing. Applying Lemma B.1 and a similar argument as in (i) we obtain the desired result. Q.E.D.

The preceding theorem shows that the optimal solution to (44) is comonotonic with $Y$, and that to (44) is anti-comonotonic with $Y$.

\section{A Choquet Maximization Problem}

Consider a general utility maximization problem involving the Choquet integral:

$$
\begin{array}{ll}
\text { Maximize } & V_{1}(X)=\int_{0}^{+\infty} T(P\{u(X)>y\}) d y \\
\text { subject to } & E[\xi X]=a, \quad X \geq 0
\end{array}
$$


where $\xi$ is a given strictly positive random variable, with no atom and whose distribution function is $F_{\xi}(\cdot), a \geq 0, T:[0,1] \mapsto[0,1]$ is a strictly increasing, differentiable function with $T(0)=0, T(1)=$ 1 , and $u(\cdot)$ is a strictly concave, strictly increasing, twice differentiable function with $u(0)=0$, $u^{\prime}(0)=+\infty, u^{\prime}(+\infty)=0$.

The case $a=0$ is trivial, where $X^{*}=0$ is the only feasible, and hence optimal, solution. So we assume $a>0$ in what follows. The difficulty with (46) is that it is a non-convex optimization problem with a constraint; thus the normal technique like Lagrange multiplier does not apply directly. The approach we develop here is to change the decision variable and turn the problem into a convex problem through a series of transformations. To start with, we have the following lemma.

Lemma C.1 If Problem (46) admits an optimal solution $X^{*}$ whose distribution function is $G(\cdot)$, then $X^{*}=G^{-1}\left(1-F_{\xi}(\xi)\right)$, a.s..

Proof: Since $a>0, G(t) \not \equiv 1$. Denote $\bar{X}:=G^{-1}\left(1-F_{\xi}(\xi)\right)$. Notice that $1-F_{\xi}(\xi) \sim U(0,1)$; thus $\bar{X}$ has the same distribution as $X^{*}$ and $E[\xi \bar{X}]>0$.

If $X^{*}=\bar{X}$ a.s. is not true, then it follows from the uniqueness result in Theorem B.1 that $E[\xi \bar{X}]<E\left[\xi X^{*}\right]=a$. Define $X_{1}:=k \bar{X}$, where $k:=a / E[\xi \bar{X}]>1$. Then $X_{1}$ is feasible for (46), and $V_{1}\left(X_{1}\right)>V_{1}(\bar{X})=V_{1}\left(X^{*}\right)$, which contradicts the optimality of $X^{*}$.

Q.E.D.

Lemma C.1 implies that an optimal solution to (46), if it exists, must be anti-comonotonic with $\xi$.

Denote $Z:=1-F_{\xi}(\xi)$. Then $Z$ follows $U(0,1)$, and $\xi=F_{\xi}^{-1}(1-Z)$, a.s., thanks to $\xi$ being atomless. Lemma C.1 suggests that in order to solve (46) one needs only to seek among random variables in the form $G^{-1}(Z)$, where $G$ is the distribution function of a nonnegative random variable [i.e., $G$ is non-decreasing, càdlàg, with $G(0-)=0, G(+\infty)=1$ ]. Motivated by this observation, we introduce the following problem

$$
\begin{array}{ll}
\text { Maximize } & v_{1}(G):=\int_{0}^{+\infty} T\left(P\left\{u\left(G^{-1}(Z)\right)>t\right\}\right) d t \\
\text { subject to } & \left\{\begin{array}{l}
E\left[G^{-1}(Z) F_{\xi}^{-1}(1-Z)\right]=a, \\
G \text { is the distribution function of a nonnegative random variable. }
\end{array}\right.
\end{array}
$$

The following result, which is straightforward in view of Lemma C.1, stipulates that Problem (47) is equivalent to Problem (46).

Proposition C.1 If $G^{*}$ is optimal for (47), then $X^{*}:=\left(G^{*}\right)^{-1}(Z)$ is optimal for (46). Conversely, if $X^{*}$ is optimal for (46), then its distribution function $G^{*}$ is optimal for (47) and $X^{*}=$ $\left(G^{*}\right)^{-1}(Z)$, a.s..

Now we turn to Problem (47). Denoting $\bar{T}(x):=T(1-x), x \in[0,1]$, and $\bar{u}:=\sup _{x \in \mathbf{R}^{+}} u(x)$, we have

$$
\begin{aligned}
v_{1}(G) & =\int_{0}^{\bar{u}} \bar{T}\left(P\left\{u\left(G^{-1}(Z)\right) \leq y\right\}\right) d y=\int_{0}^{\bar{u}} \bar{T}\left(P\left\{Z \leq G\left(u^{-1}(y)\right)\right\}\right) d y \\
& =\int_{0}^{\bar{u}} \bar{T}\left(G\left(u^{-1}(y)\right)\right) d y=\int_{0}^{1} u\left(G^{-1}\left(\bar{T}^{-1}(t)\right)\right) d t \\
& =-\int_{0}^{1} u\left(G^{-1}(s)\right) \bar{T}^{\prime}(s) d s=\int_{0}^{1} u\left(G^{-1}(s)\right) T^{\prime}(1-s) d s \\
& =E\left[u\left(G^{-1}(Z)\right) T^{\prime}(1-Z)\right] .
\end{aligned}
$$


Denoting

$$
\Gamma:=\left\{g:[0,1) \mapsto \mathbb{R}^{+} \text {is non-decreasing, left continuous, with } g(0)=0\right\},
$$

and considering $g=G^{-1}$, we can rewrite Problem (47) into

$$
\begin{array}{ll}
\text { Maximize } & \bar{v}_{1}(g):=E\left[u(g(Z)) T^{\prime}(1-Z)\right] \\
\text { subject to } & E\left[g(Z) F_{\xi}^{-1}(1-Z)\right]=a, \quad g \in \Gamma .
\end{array}
$$

Some remarks on the set $\Gamma$ are in order. Since any given $g \in \Gamma$ is left continuous, we can always extend it to a map from $[0,1]$ to $\mathbb{R}^{+} \cup\{+\infty\}$ by setting $g(1):=g(1-)$. It is easy to see that $g(1)<+\infty$ if and only if the corresponding random variable $\eta$ (i.e., $\eta$ is such a random variable whose distribution function has an inverse identical to $g$ ) is almost surely bounded from above.

Since $T^{\prime}(\cdot)>0$ and $u(\cdot)$ is concave, the objective functional of (48) is now concave in $g$. On the other hand, the constraint functional $E\left[g(Z) F_{\xi}^{-1}(1-Z)\right]$ is linear in $g$. Hence we can use the Lagrange method to remove this linear constraint as follows. For a given $\lambda \in \mathbb{R}$,

$$
\begin{array}{ll}
\text { Maximize } & \tilde{v}_{1}^{\lambda}(g):=E\left[u(g(Z)) T^{\prime}(1-Z)-\lambda g(Z) F_{\xi}^{-1}(1-Z)\right] \\
\text { subject to } \quad g \in \Gamma,
\end{array}
$$

and then determine $\lambda$ via the original linear constraint.

Although Problem (49) is a convex optimization problem in $g$, it has an implicit constraint that $g$ be non-decreasing; hence is very complex. Let us ignore this constraint for the moment. For each fixed $z \in(0,1)$ we maximize $u(g(z)) T^{\prime}(1-z)-\lambda g(z) F_{\xi}^{-1}(1-z)$ over $g(z) \in \mathbb{R}^{+}$. The zero-derivative condition gives $g(z)=\left(u^{\prime}\right)^{-1}\left(\lambda F_{\xi}^{-1}(1-z) / T^{\prime}(1-z)\right)$. Now, if $F_{\xi}^{-1}(z) / T^{\prime}(z)$ happens to be nondecreasing in $z \in(0,1]$, then $g(z)$ is non-decreasing in $z \in[0,1)$ and, hence, it solves (49). On the other hand, if $F_{\xi}^{-1}(z) / T^{\prime}(z)$ is not non-decreasing, then it remains an open problem to express explicitly the optimal solution to (49).

Denote $R_{u}(x):=-\frac{x u^{\prime \prime}(x)}{u^{\prime}(x)}, x>0$, which is the Arrow-Pratt index of relative risk aversion of the utility function $u(\cdot)$.

Proposition C.2 Assume that $F_{\xi}^{-1}(z) / T^{\prime}(z)$ is non-decreasing in $z \in(0,1]$ and $\liminf _{x \rightarrow+\infty} R_{u}(x)>0$. Then the following claims are equivalent:

(i) Problem (48) is well-posed for any $a>0$.

(ii) Problem (48) admits a unique optimal solution for any $a>0$.

(iii) $E\left[u\left(\left(u^{\prime}\right)^{-1}\left(\frac{\xi}{T^{\prime}\left(F_{\xi}(\xi)\right)}\right)\right) T^{\prime}\left(F_{\xi}(\xi)\right)\right]<+\infty$.

(iv) $E\left[u\left(\left(u^{\prime}\right)^{-1}\left(\frac{\lambda \xi}{T^{\prime}\left(F_{\xi}(\xi)\right)}\right)\right) T^{\prime}\left(F_{\xi}(\xi)\right)\right]<+\infty \forall \lambda>0$.

Furthermore, when one of the above (i)-(iv) holds, the optimal solution to (48) is

$$
g^{*}(x) \equiv\left(G^{*}\right)^{-1}(x)=\left(u^{\prime}\right)^{-1}\left(\frac{\lambda F_{\xi}^{-1}(1-x)}{T^{\prime}(1-x)}\right), x \in[0,1),
$$

where $\lambda>0$ is the one satisfying $E\left[\left(G^{*}\right)^{-1}\left(1-F_{\xi}(\xi)\right) \xi\right]=a$. 
Proof: Since $T^{\prime}(1-Z)>0$ and $E\left[T^{\prime}(1-Z)\right]=\int_{0}^{1} T^{\prime}(x) d x=T(1)-T(0)=1$, we can define a new probability measure $\tilde{P}$ whose expectation $\tilde{E}(X):=E\left[T^{\prime}(1-Z) X\right]$.

Denote $\zeta:=\frac{F_{\xi}^{-1}(1-Z)}{T^{\prime}(1-Z)} \equiv \frac{\xi}{T^{\prime}\left(F_{\xi}(\xi)\right)}$. Then $\zeta>0$ a.s.. Rewrite Problem (48) in terms of the probability measure $\tilde{P}$ as follows

$$
\begin{array}{ll}
\text { Maximize } & \bar{v}_{1}(g):=\tilde{E}[u(g(Z))] \\
\text { subject to } & \tilde{E}[\zeta g(Z)]=a, \quad g \in \Gamma .
\end{array}
$$

By Jin, Xu and Zhou (2007, Theorem 6) and the fact that $g^{*}(x)=\left(u^{\prime}\right)^{-1}\left(\frac{\lambda F_{\xi}^{-1}(1-x)}{T^{\prime}(1-x)}\right)$ is automatically non-decreasing in $x$, we get the desired result.

We now summarize all the results above in the following theorem.

Theorem C.1 Assume that $F_{\xi}^{-1}(z) / T^{\prime}(z)$ is non-decreasing in $z \in(0,1]$ and $\liminf \operatorname{in}_{x \rightarrow+\infty} R_{u}(x)>$ 0 . Define $X(\lambda):=\left(u^{\prime}\right)^{-1}\left(\frac{\lambda \xi}{T^{\prime}\left(F_{\xi}(\xi)\right)}\right)$ for $\lambda>0$. If $V_{1}(X(1))<+\infty$, then $X(\lambda)$ is an optimal solution for Problem (46), where $\lambda$ is the one satisfying $E[\xi X(\lambda)]=a$. If $V_{1}(X(1))=+\infty$, then Problem (46) is ill-posed.

To conclude this subsection, we state a necessary condition of optimality for Problem (46), which is useful in solving Problem (13) in Step 2.

Lemma C.2 If $g$ is optimal for (49), then either $g \equiv 0$ or $g(x)>0 \forall x>0$.

Proof: Suppose $g \not \equiv 0$. We now show that $g(x)>0 \forall x>0$. If not, define $\delta:=\inf \{x>0$ : $g(x)>0\}$. Then $0<\delta<1$, and $g(x)=0 \forall x \in[0, \delta]$.

For any $y>0$, let $\epsilon(y):=\inf \{x>0: g(\delta+x)>y\}$ and

$$
g_{y}(x):= \begin{cases}0, & x \in[0, \delta / 2], \\ y, & x \in(\delta / 2, \delta+\epsilon(y)], \\ g(x), & x \in(\delta+\epsilon(y), 1) .\end{cases}
$$

Then

$$
\begin{aligned}
& E\left[u\left(g_{y}(Z)\right) T^{\prime}(1-Z)-\lambda g_{y}(Z) F_{\xi}^{-1}(1-Z)\right]-E\left[u(g(Z)) T^{\prime}(1-Z)-\lambda g(Z) F_{\xi}^{-1}(1-Z)\right] \\
= & \int_{\delta / 2}^{\delta+\epsilon(y)}\left[u(y) T^{\prime}(1-x)-\lambda y F_{\xi}^{-1}(1-x)\right] d x-\int_{\delta}^{\delta+\epsilon(y)}\left[u(g(x)) T^{\prime}(1-x)-\lambda g(x) F_{\xi}^{-1}(1-x)\right] d x \\
\geq & \int_{\delta / 2}^{\delta+\epsilon(y)}\left[u(y) T^{\prime}(1-x)-\lambda y F_{\xi}^{-1}(1-x)\right] d x-\int_{\delta}^{\delta+\epsilon(y)} u(g(x)) T^{\prime}(1-x) d x \\
\geq & u(y) \int_{\delta / 2}^{\delta} T^{\prime}(1-x) d x-\lambda y \int_{\delta / 2}^{\delta+\epsilon(y)} F_{\xi}^{-1}(1-x) d x \\
= & y\left[\frac{u(y)}{y}(T(1-\delta / 2)-T(1-\delta))-\lambda \int_{\delta / 2}^{\delta+\epsilon(y)} F_{\xi}^{-1}(1-x) d x\right] .
\end{aligned}
$$


Since $\frac{u(y)}{y} \rightarrow+\infty$ as $y \rightarrow 0$ and $T(1-\delta / 2)-T(1-\delta)>0$, we have $\frac{u(y)}{y}(T(1-\delta / 2)-T(1-\delta)) \rightarrow$ $+\infty$ as $y \rightarrow 0$. On the other hand, $\epsilon(y) \rightarrow 0$ as $y \rightarrow 0$; hence

$$
\int_{\delta / 2}^{\delta+\epsilon(y)} F_{\xi}^{-1}(1-x) d x \leq(\epsilon(y)+\delta / 2) F_{\xi}^{-1}(1-\delta / 2) \rightarrow \delta / 2 F_{\xi}^{-1}(1-\delta / 2) \quad \text { as } y \rightarrow 0 .
$$

Consequently,

$$
\frac{u(y)}{y}(T(1-\delta / 2)-T(1-\delta))-\lambda \int_{\delta / 2}^{\delta+\epsilon(y)} F_{\xi}^{-1}(1-x) d x \rightarrow+\infty \quad \text { as } y \rightarrow 0 .
$$

Fix $y>0$ sufficiently small so that the left hand side of the above is no less than 1 . Then

$$
E\left[u\left(g_{y}(Z)\right) T^{\prime}(1-Z)-\lambda g_{y}(Z) F_{\xi}^{-1}(1-Z)\right]-E\left[u(g(Z)) T^{\prime}(1-Z)-\lambda g(Z) F_{\xi}^{-1}(1-Z)\right] \geq y>0,
$$

which implies that $g_{y}$ is strictly better than $g$ for (49).

Q.E.D.

TheOREM C.2 If $X^{*}$ is an optimal solution for (46) with some $a>0$, then $P\left(X^{*}=0\right)=0$.

Proof: Proposition C.1 implies that the distribution function $G^{*}$ of $X^{*}$ is optimal for (47). By Lagrange method there exists $\lambda \geq 0$ such that $\left(G^{*}\right)^{-1}$ is optimal for (49). Since $a>0,\left(G^{*}\right)^{-1} \not \equiv 0$. It follows then from Lemma C. 2 that $\left(G^{*}\right)^{-1}(x)>0 \forall x>0$, or $G^{*}(0)=0$.

Q.E.D.

So an optimal solution to (46) with a positive initial budget is positive almost surely.

\section{A Choquet Minimization Problem}

Consider a general utility minimization problem involving the Choquet integral:

$$
\begin{array}{ll}
\text { Minimize } & V_{2}(X):=\int_{0}^{+\infty} T(P\{u(X)>y\}) d y \\
\text { subject to } & E[\xi X]=a, \quad X \geq 0,
\end{array}
$$

where $\xi, a, T(\cdot)$ satisfy the same assumptions as those with Problem (46), and $u(\cdot)$ is strictly increasing, concave with $u(0)=0$.

It is easy to see that (51) always admits feasible solutions (e.g., $X=x \mathbf{1}_{\xi \leq \xi_{0}}$ is feasible with appropriate $x \in \mathbb{R}, \xi_{0} \in \mathbb{R}$ ); hence the optimal value of (51) is a finite nonnegative number.

In view of Theorem B.1, a similar argument to that in Appendix C reveals that the optimal solution $X^{*}$ to $(51)$ must be in the form of $G^{-1}\left(F_{\xi}(\xi)\right)$ for some distribution function $G(\cdot)$, which can be determined by the following problem

$$
\begin{array}{ll}
\text { Minimize } & v_{2}(G):=\int_{0}^{+\infty} T\left(P\left\{u\left(G^{-1}(Z)\right)>y\right\}\right) d y \\
\text { subject to } & \left\{\begin{array}{l}
E\left[G^{-1}(Z) F_{\xi}^{-1}(Z)\right]=a, \\
G \text { is the distribution function of a nonnegative random variable, }
\end{array}\right.
\end{array}
$$

where $Z:=F_{\xi}(\xi)$.

Proposition D.1 If $G^{*}$ is optimal for (52), then $X^{*}:=\left(G^{*}\right)^{-1}(Z)$ is optimal for (51). Conversely, if $X^{*}$ is optimal for (51), then its distribution function $G^{*}$ is optimal for (52) and $X^{*}=$ $\left(G^{*}\right)^{-1}(Z)$, a.s.. 
By the same calculation as in Appendix C, we have $v_{2}(G)=E\left[u\left(G^{-1}(Z)\right) T^{\prime}(1-Z)\right]$. Denoting $g=G^{-1}$, Problem (52) can be rewritten as

$$
\begin{array}{ll}
\text { Minimize } & \bar{v}_{2}(g):=E\left[u(g(Z)) T^{\prime}(1-Z)\right] \\
\text { subject to } & E\left[g(Z) F_{\xi}^{-1}(Z)\right]=a, \quad g \in \Gamma .
\end{array}
$$

Since the objective of the above problem is to minimize a concave functional, its solution must have a very different structure compared with Problem (46), which in turn requires a completely different technique to obtain. Specifically, the solution should be a "corner point solution" (in the terminology of linear program). The question is how to characterize such a corner point solution in the present setting.

Proposition D.2 Assume that $u(\cdot)$ is strictly concave at 0 . Then the optimal solution for Problem (53), if it exists, must be in the form $g(t)=q(b) \mathbf{1}_{(b, 1)}(t), t \in[0,1)$, with some $b \in[0,1)$ and $q(b):=\frac{a}{E\left[F_{\xi}^{-1}(Z) \mathbf{1}_{(b, 1)(Z)}\right]}$.

Proof: Denote $f(\cdot):=F_{\xi}^{-1}(\cdot)$ for notational convenience. We assume $a>0$ (otherwise the result holds trivially). If $g$ is an optimal solution to $(53)$, then $g \not \equiv 0$. Fix $t_{1} \in(0,1)$ such that $g\left(t_{1}\right)>0$. Define $k:=\frac{\int_{0}^{1} g(t) f(t) d t}{\int_{0}^{t_{1}} g(t) f(t) d t+g\left(t_{1}\right) \int_{t_{1}}^{1} f(t) d t}$, and

$$
\bar{g}(t):= \begin{cases}k g(t), & \text { if } t \in\left[0, t_{1}\right] \\ k g\left(t_{1}\right) & \text { if } t \in\left(t_{1}, 1\right) .\end{cases}
$$

Then $\bar{g}(\cdot) \in \Gamma$, and $\int_{0}^{1} \bar{g}(t) f(t) d t=k \int_{0}^{t_{1}} g(t) f(t) d t+k g\left(t_{1}\right) \int_{t_{1}}^{1} f(t) d t=\int_{0}^{1} g(t) f(t) d t$, implying that $\bar{g}(\cdot)$ is feasible for (53). We now claim that $g(t)=g\left(t_{1}\right)$, a.e. $t \in\left(t_{1}, 1\right)$. Indeed, if this is not true, then $k>1$. Define $\lambda:=1-1 / k \in(0,1)$ and $\tilde{g}(t):=\frac{g(t)-g\left(t_{1}\right)}{\lambda} \mathbf{1}_{t>t_{1}}, t \in[0,1)$. Then

$$
(1-\lambda) \bar{g}(t)+\lambda \tilde{g}(t)=g(t) \forall t \in[0,1) .
$$

It follows from the concavity of $u(\cdot)$ that $\bar{v}_{2}(g) \geq(1-\lambda) \bar{v}_{2}(\bar{g})+\lambda \bar{v}_{2}(\tilde{g})$, and the equality holds only if

$$
u(g(t))=(1-\lambda) u(\bar{g}(t))+\lambda u(\tilde{g}(t)) \text {, a.e. } t \in(0,1) .
$$

Owing to the optimality of $g$, the above equality does hold. However, the equality when $t \leq t_{1}$ implies that $u(\cdot)$ is not strictly concave at 0 , which is a contradiction.

Denote $b:=\inf \{t \geq 0: g(t)>0\}$. The preceding analysis shows that $g(t)=k \mathbf{1}_{t>b}$ for some $k \in \mathbb{R}^{+}$. The feasibility of $g(\cdot)$ determines $k \equiv q(b)=\frac{a}{E\left[F_{\xi}^{-1}(Z) \mathbf{1}_{(b, 1)(Z)}\right]}$. Q.E.D.

By left-continuity one can extend the optimal $g$ described in Proposition D.2 to $[0,1]$ by defining $g(1):=q(b)$. Moreover, since $g(t)$ is uniformly bounded in $t \in[0,1]$, it follows from Proposition D.1 that any optimal solution $X^{*}$ to $(51)$ can be represented as $X^{*}=g(Z)$, hence must be uniformly bounded from above.

Proposition D.2 suggests that we only need to find an optimal number $b \in[0,1)$ so as to solve Problem (53), which motivates the introduction of the following problem

$$
\begin{array}{ll}
\text { Minimize } & \tilde{v}_{2}(b):=E\left[u(g(Z)) T^{\prime}(1-Z)\right] \\
\text { subject to } & g(\cdot)=\frac{a}{E\left[F_{\xi}^{-1}(Z) \mathbf{1}_{(b, 1]}(Z)\right]} \mathbf{1}_{(b, 1]}(\cdot), \quad 0 \leq b<1
\end{array}
$$


Proposition D.3 Problems (53) and (55) have the same infimum values.

Proof: Denote by $\alpha$ and $\beta$ the infimum values of Problems (53) and (55) respectively. Clearly $\alpha \leq \beta$. If the opposite inequality is false, then there is a feasible solution $g$ for (53) such that $\bar{v}_{2}(g)<\beta$.

For any $s \in[0,1)$, define $k(s)=\frac{\int_{0}^{1} g(t) f(t) d t}{\int_{0}^{s} g(t) f(t) d t+g(s) \int_{s}^{1} f(t) d t} \geq 1$, where $f(\cdot):=F_{\xi}^{-1}(\cdot)$. Then $\lim _{s \rightarrow 1} k(s)=1$. Define

$$
H_{s}(t):= \begin{cases}k(s) g(t), & t \in[0, s] \\ k(s) g(s), & t \in(s, 1) .\end{cases}
$$

As shown in the proof of Proposition D.2, $H_{s}(\cdot)$ is feasible for (53), and

$$
\begin{aligned}
\bar{v}_{2}\left(H_{s}\right) & \leq \int_{0}^{1} u(k(s) g(t)) T^{\prime}(1-t) d t \\
& \leq \int_{0}^{1} k(s) u(g(t)) T^{\prime}(1-t) d t \rightarrow \bar{v}_{2}(g), \text { as } s \rightarrow 1 .
\end{aligned}
$$

Therefore there exists $s \in[0,1)$, which we now fix, such that $v_{2}\left(H_{s}\right)<\beta$. For any nonnegative integer $n$, define $a(n, k):=\frac{\int_{(k-1) / 2^{n}}^{k / 2^{n}} H_{s}(t) f(t) d t}{\int_{(k-1) / 2^{n}}^{k n} f(t) d t}$, for any $k=1, \cdots, 2^{n}$. It is clear that $H_{s}\left((k-1) / 2^{n}\right) \leq$ $a(n, k) \leq H_{s}\left(k / 2^{n}\right)$. Define

$$
g_{n}(t):=\sum_{k=1}^{2^{n}} a(n, k) \mathbf{1}_{\left((k-1) / 2^{n}, k / 2^{n}\right]}(t), \quad t \in[0,1) .
$$

Clearly $g_{n} \in \Gamma$, and $\int_{0}^{1} g_{n}(t) f(t) d t=\int_{0}^{1} H_{s}(t) f(t) d t=a$, implying that $g_{n}$ is feasible for (53) for each $n$. Furthermore, $g_{n}(t) \rightarrow H_{s}(t) \quad \forall t$ and $0 \leq g_{n}(t) \leq k(s) g(s) \quad \forall t$, which leads to $\bar{v}_{2}\left(g_{n}\right) \rightarrow \bar{v}_{2}\left(H_{s}\right)$. So there exists $n$ such that $\bar{v}_{2}\left(g_{n}\right)<\beta$.

Because $g_{n}(\cdot)$ is a left continuous and non-decreasing step function, we can rewrite it as

$$
g_{n}(t)=\sum_{k=1}^{m} a_{k-1} \mathbf{1}_{\left(t_{k-1}, t_{k}\right]}(t)
$$

with $0=t_{0}<t_{1}<\cdots<t_{m}=1,0=a_{0}<a_{1}<a_{2}<\cdots<a_{m}<+\infty$. Denote $\lambda_{k}:=\frac{a_{k}-a_{k-1}}{q\left(t_{k}\right)}$, $k=1,2, \cdots, m$, where $q(\cdot)$ is defined in Proposition D.2. Then for any $t \in(0,1)$,

$$
g_{n}(t)=\sum_{k=1}^{m} a_{k-1} \mathbf{1}_{\left(t_{k-1}, t_{k}\right]}(t)=\sum_{k=1}^{m}\left(a_{k}-a_{k-1}\right) \mathbf{1}_{\left(t_{k}, 1\right]}=\sum_{k=1}^{m} \lambda_{k} J_{t_{k}}(t),
$$

where $J_{t_{k}}(t):=q\left(t_{k}\right) \mathbf{1}_{\left(t_{k}, 1\right]}$. Since

$$
a \equiv \int_{0}^{1} g_{n}(t) f(t) d t=\sum_{k=1}^{m} \lambda_{k} \int_{0}^{1} J_{t_{k}}(t) f(t) d t=\sum_{k=1}^{m} \lambda_{k} a,
$$

we conclude that $\sum_{k=1}^{m} \lambda_{k}=1$, which means that $g_{n}$ is a convex combination of $J_{t_{k}}$. It follows from the concavity of $u(\cdot)$ that there exists $k$ such that $\bar{v}_{2}\left(J_{t_{k}}\right) \leq \bar{v}_{2}\left(g_{n}\right)$, which contradicts the conclusion that $\bar{v}_{2}\left(g_{n}\right)<\beta \leq \bar{v}_{2}\left(J_{t_{k}}\right)$.

Q.E.D.

Summarizing, we have the following result. 
Theorem D.1 Problems (51) and (55) have the same infimum values. If, in addition, $u(\cdot)$ is strictly concave at 0 , then (51) admits an optimal solution if and only if the following problem

$$
\min _{0 \leq c<\operatorname{esssup}} u\left(\frac{a}{E\left[\xi \mathbf{1}_{\xi>c}\right]}\right) T(P(\xi>c))
$$

admits an optimal solution $c^{*}$, in which case the optimal solution to (51) is $X^{*}=\frac{a}{E\left[\xi \mathbf{1}_{\xi>c^{*}}\right]} \mathbf{1}_{\xi>c^{*}}$.

Proof: The first conclusion follows from Proposition D.3. For the second conclusion, we rewrite the objective functional of $(55)$ as

$$
\tilde{v}_{2}(b)=E\left[u\left(q(b) \mathbf{1}_{(b, 1]}(Z)\right) T^{\prime}(1-Z)\right]=\int_{b}^{1} u(q(b)) T^{\prime}(1-t) d t=u(q(b)) T(1-b),
$$

where $b \in[0,1)$. Now let $c:=F_{\xi}^{-1}(b) \in[0, \operatorname{esssup} \xi)$. Then

$$
\tilde{v}_{2}(b)=u(q(b)) T(1-b)=u\left(\frac{a}{E\left[\xi \mathbf{1}_{\xi>c}\right]}\right) T(P(\xi>c))
$$

and the desired results are straightforward in view of Theorem D.2.

Q.E.D.

\section{E Replicating a Binary Option}

In this subsection, we want to find a portfolio replicating the contingent claim $\rho^{\alpha} \mathbf{1}_{\rho \in\left(c_{1}, c_{2}\right)}$, where $0 \leq c_{1}<c_{2} \leq+\infty, \alpha \in \mathbb{R}$, and $\rho=\rho(T)$ with

$$
\rho(t):=\exp \left\{-\left(r+\frac{1}{2}|\theta|^{2}\right) t-\theta^{\prime} W(t)\right\}, \quad 0 \leq t \leq T .
$$

The claim resembles the payoff of a binary (or digital) option, except that $\rho$ does not correspond to any underlying stock [although it is indeed the terminal wealth of a mutual fund; see Bielecki et al. (2005, Remark 7.3), for details].

Let $\psi(\cdot)$ and $N(\cdot)$ be the density function and distribution function of the standard normal distribution respectively. Recall that $\rho(t, T):=\rho(T) / \rho(t)$ conditional on $\mathcal{F}_{t}$ follows a lognormal distribution with parameters $\left(\mu_{t}, \sigma_{t}^{2}\right)$ given by $(41)$.

THEOREM E.1 If $c_{2}<+\infty$, then the wealth-portfolio pair replicating $\rho^{\alpha} \mathbf{1}_{\rho \in\left(c_{1}, c_{2}\right)}$ is

$$
\begin{aligned}
& x(t)=\frac{\rho(t)^{\alpha}}{\sigma_{t}} \int_{c_{1} / \rho(t)}^{c_{2} / \rho(t)} y^{\alpha} \psi\left(\frac{\ln y-\mu_{t}}{\sigma_{t}}\right) d y, \\
& \pi(t)=-\left[\alpha x(t)-\frac{1}{\sigma_{t} \rho(t)}\left(c_{2}^{\alpha+1} \psi\left(\frac{\ln c_{2}-\mu_{t}-\ln \rho(t)}{\sigma_{t}}\right)-c_{1}^{\alpha+1} \psi\left(\frac{\ln c_{1}-\mu_{t}-\ln \rho(t)}{\sigma_{t}}\right)\right)\right]\left(\sigma \sigma^{\prime}\right)^{-1} B .
\end{aligned}
$$

If $c_{2}=+\infty$, then the corresponding replicating pair is

$$
\begin{aligned}
& x(t)=\frac{\rho(t)^{\alpha}}{\sigma_{t}} \int_{c_{1} / \rho(t)}^{+\infty} y^{\alpha} \psi\left(\frac{\ln y-\mu_{t}}{\sigma_{t}}\right) d y, \\
& \pi(t)=-\left[\alpha x(t)+\frac{1}{\sigma_{t} \rho(t)} c_{1}^{\alpha+1} \psi\left(\frac{\ln c_{1}-\mu_{t}-\ln \rho(t)}{\sigma_{t}}\right)\right]\left(\sigma \sigma^{\prime}\right)^{-1} B .
\end{aligned}
$$


Proof: When $c_{2}<+\infty$, the replicating wealth process is

$$
\begin{aligned}
x(t) & =E\left[\rho(t, T) \rho(T)^{\alpha} \mathbf{1}_{\rho(T) \in\left(c_{1}, c_{2}\right)} \mid \mathcal{F}_{t}\right] \\
& =\rho(t)^{\alpha} E\left[\rho(t, T)^{\alpha+1} \mathbf{1}_{\rho(t, T) \in\left(c_{1} / \rho(t), c_{2} / \rho(t)\right)} \mid \mathcal{F}_{t}\right] \\
& =\rho(t)^{\alpha} \int_{c_{1} / \rho(t)}^{c_{2} / \rho(t)} y^{\alpha+1} d N\left(\frac{\ln y-\mu_{t}}{\sigma_{t}}\right) \\
& =\frac{\rho(t)^{\alpha}}{\sigma_{t}} \int_{c_{1} / \rho(t)}^{c_{2} / \rho(t)} y^{\alpha} \psi\left(\frac{\ln y-\mu_{t}}{\sigma_{t}}\right) d y=f(t, \rho(t)),
\end{aligned}
$$

where $f(t, \rho):=\frac{\rho^{\alpha}}{\sigma_{t}} \int_{c_{1} / \rho}^{c_{2} / \rho} y^{\alpha} \psi\left(\frac{\ln y-\mu_{t}}{\sigma_{t}}\right) d y$. It is well known that the replicating portfolio is

$$
\pi(t)=-\left(\sigma \sigma^{\prime}\right)^{-1} B \frac{\partial f(t, \rho(t))}{\partial \rho} \rho(t)
$$

see, e.g., Bielecki et al. (2005, Eq. (7.6)). Now we calculate

$$
\begin{aligned}
\frac{\partial f(t, \rho)}{\partial \rho}= & \frac{\alpha \rho^{\alpha-1}}{\sigma_{t}} \int_{c_{1} / \rho}^{c_{2} / \rho} y^{\alpha} \psi\left(\frac{\ln y-\mu_{t}}{\sigma_{t}}\right) d y \\
& +\frac{\rho^{\alpha}}{\sigma_{t}}\left[\left(\frac{c_{2}}{\rho}\right)^{\alpha} \psi\left(\frac{\ln c_{2}-\mu_{t}-\ln \rho}{\sigma_{t}}\right) \frac{-c_{2}}{\rho^{2}}-\left(\frac{c_{1}}{\rho}\right)^{\alpha} \psi\left(\frac{\ln c_{1}-\mu_{t}-\ln \rho}{\sigma_{t}}\right) \frac{-c_{1}}{\rho^{2}}\right] \\
= & \frac{\alpha x(t)}{\rho}-\frac{1}{\sigma_{t} \rho^{2}}\left[c_{2}^{\alpha+1} \psi\left(\frac{\ln c_{2}-\mu_{t}-\ln \rho}{\sigma_{t}}\right)-c_{1}^{\alpha+1} \psi\left(\frac{\ln c_{1}-\mu_{t}-\ln \rho}{\sigma_{t}}\right)\right] .
\end{aligned}
$$

Plugging in (56) we get the desired result.

The case with $c_{2}=+\infty$ can be dealt with similarly (in fact more easily).

Q.E.D.

\section{References}

M. Allais (1953), Le comportement de l'homme rationnel devant le risque, critique des postulats et axiomes de l'ecole americaine, Econometrica, 21, pp. 503-546.

G.W. Bassett, JR., R. Koenker and G. Kordas (2004), Pessimistic portfolio allocation and Choquet expected utility, J. Financial Econometrics, 2, pp. 477-492.

S. Benartzi And R. Thaler (1995), Myopic loss aversion and the equity premium puzzle, Quart. J. Econ., 110 , pp. 73-92.

A.B. Berkelaar, R. Kouwenberg and T. Post (2004), Optimal portfolio choice under loss aversion, Rev. Econ. Stat., 86, pp. 973-987.

T.R. Bielecki, H. Jin, S.R. Pliska And X.Y. Zhou (2005), Continuous-time mean-variance portfolio selection with bankruptcy prohibition, Math. Finance, 15, pp. 213-244.

G. Choquet (1953/54), Theory of capacities, Ann. de l'Inst. Fourier, 5, pp. 131-295.

J.C. Cox And C.-F. Huang (1989), Optimal consumption and portfolio policies when asset prices follow a diffusion process, J. Econom. Theory, 49, 33-83.

E. De Giorgi And T. Post (2005), Second order stochastic dominance, reward-risk portfolio selection and CAPM, J. Financial Quant. Anal., to appear.

D. Denneberg (1994), Non-Additive Measure and Integral, Kluwer, Dordrecht.

D. Duffie (1996), Dynamic Asset Pricing Theory, 2nd Edition, Princeton University Press, 
Princeton.

D. Ellsberg (1961), Risk, ambiguity and the Savage axioms, Quart. J. Econom., 75, pp. 643669.

P.C. Fishburn (1998), Nonlinear Preference and Utility Theory, The John Hopkins University Press, Baltimore.

H. Föllmer And A. Schied (2002), Stochastic Finance: An Introduction in Discrete Time, Walter de Gruyter, Berlin.

M. Friedman and L.J. Savage (1948), The utility analysis of choices involving risk, J. Political Economy, 56, pp. 279-304.

F.J. Gomes (2005), Portfolio choice and trading volume with loss-averse investors, J. Business, 78, pp. 675-706.

X. He And X.Y. Zhou (2007), Behavioral portfolio choice: Model, theory, and equity premium puzzle, working paper, The Chinese University of Hong Kong.

H. Jin, J.A. Yan And X.Y. Zhou (2005), Continuous-time mean-risk portfolio selection, Ann. de l'Institut Henri Poincaré: Probab. 65 Stat., 41, pp. 559-580.

H. Jin, Z. XU AND X.Y. Zhou (2007), A convex stochastic optimization problem arising from portfolio selection, Math. Finance, to appear.

D. Kahneman And A. Tversky (1979), Prospect theory: An analysis of decision under risk, Econometrica, 47, pp. 263-291.

I. Karatzas and S.E. Shreve (1998), Methods of Mathematical Finance, Springer-Verlag, New York.

R. Korn and H. Kraft (2004), On the stability of continuous-time portfolio problems with stochastic opportunity set, Math. Finance 14, pp. 403-414.

H. Levy And M. Levy (2004), Prospect theory and mean-variance analysis, Rev. Financial Studies, 17, pp. 1015-1041.

D. Li AND W.L. NG (2000), Optimal dynamic portfolio selection: Multiperiod mean-variance formulation, Math. Finance, 10, pp. 387-406.

R. Mehra And E.C. Prescott (1985), The equity premium: A puzzle, J. Monet. Econ., 15, pp. $145-161$.

J. von Neumann and O. Morgenstern (1944), Theory of Games and Economic Behavior, Princeton University Press, Princeton.

H. Shefrin and M. Statman (2000), Behavioral portfolio theory, J. Finan. Quant. Anal., 35, pp. 127-151.

A. Tversky and D. Kahneman (1992), Advances in prospect theory: Cumulative representation of uncertainty. J. Risk $\&$ Uncertainty, 5, pp. 297-323.

X.Y. Zhou And D. Li (2000), Continuous time mean-variance portfolio selection: A stochastic LQ framework, Appl. Math. \& Optim., 42, pp. 19-33. 